\title{
Metric-like Methods in Higher Spin Holography
}

\section{Charlotte Sleight*}

Université Libre de Bruxelles and International Solvay Institutes

ULB-Campus Plaine CP231, 1050 Brussels, Belgium

Max-Planck-Institut für Physik

Föhringer Ring 6, 80805 Munich, Germany

E-mail: charlotte.sleightegmail.com

These notes comprise part of the introductory lectures on Higher Spin Theories presented at the Twelfth Modave Summer School in Mathematical Physics 2016, aimed at Ph.D. students and researchers new to this topic. The focus is on the conjectured interpretation of higher-spin gauge theories on anti-de Sitter (AdS) backgrounds as holographic duals to free Conformal Field Theories.

XII Modave Summer School in Mathematical Physics

12-16 September 2016

Modave, Belgium

${ }^{*}$ Speaker. 


\section{Introduction}

The study of higher-spin gauge theories has a long history. Since the early works in the 1930's of Majorana [1], Dirac [2], Fierz [3] together with Pauli [4], and Wigner [5], by now the free propagation of higher-spin gauge fields is rather well understood (for instance see: [6-20]). On the other hand, the question of constructing consistent interactions among them is a highly non-trivial one (for a review see [21]).

One of the main motivations for studying higher-spin gauge theories is the on-going quest for a UV-complete theory of gravity. Indeed, upon the addition of higher-derivative counter-terms to the Einstein-Hilbert action, ${ }^{1}$ to avoid violations of causality at the classical level we are led to introduce an infinite tower of massive particles of spins $s>2$ [22,23]. One may then expect an underlying higher-spin symmetry principle governing the high-energy behaviour of the theory, whose spontaneous breaking would generate the lower energy spectrum of massive higher-spin states. This picture was also motivated from a String Theory perspective by Gross [24] in the 1980's.

Higher-spin gauge theories have generated an increased interest in the last two decades, owing in particular to their role in the celebrated AdS/CFT correspondence [25-27]. Theories of higherspin gauge fields on anti-de Sitter backgrounds have been conjectured to be dual to very simple, free, Conformal Field Theories [28-31]. This has the potential to provide a powerful framework to acquire a deeper understanding of AdS/CFT, and also into higher-spin gauge theories themselves.

In these notes we will focus on the latter. In particular, we review some recent efforts [32-35] aimed at using holography to study interactions of higher-spin particles. ${ }^{2}$ To this end, we introduce some useful tools for computing tree-level amplitudes in AdS involving fields of arbitrary integer spin. These are underpinned by the so-called ambient space formalism introduced by Dirac in the 1930's [39], in which anti-de Sitter space is viewed as a one-sheeted hyperboloid embedded in a higher-dimensional flat space. In order to be self-contained, we also review relevant aspects of the AdS/CFT correspondence and the basics of higher-spin particles on AdS.

\section{Higher Spin Particles in AdS}

\subsection{The AdS Geometry and Isometry Group}

From the holographic view point taken in these lectures, we are interested in particles propagating on a $(d+1)$-dimensional anti-de Sitter $\left(\mathrm{AdS}_{d+1}\right)$ background, which can be regarded as the hyperboloid ${ }^{3}$

$$
-X_{0}^{2}-X_{d+1}^{2}+\sum_{i=1}^{d} X_{i}^{2}=-R^{2}
$$

\footnotetext{
${ }^{1}$ For example, at one-loop one includes the Gauss-Bonnet term.

${ }^{2}$ For related works by other authors see [36-38].

${ }^{3}$ The length scale $R$ is known as the AdS radius, which is related to the cosmological constant $\Lambda$ via

$$
\Lambda=-\frac{d(d-1)}{2 R^{2}}<0 .
$$
}


embedded in an ambient $(d+2)$-dimensional flat space time with metric

$$
d s^{2}=\eta_{A B} d X^{A} d X^{B}=-d X_{0}^{2}-d X_{d+1}^{2}+\sum_{i=1}^{d} d X_{i}^{2},
$$

where $\eta_{A B}=\operatorname{diag}(-++\cdots+-)$ and $A, B=0,1, \ldots, d+1$. More concretely, denoting the intrinsic co-ordinates on our hyperboloid (1.2) by $x^{\mu}$ (with $\mu=0, \ldots, d$ ), we are making a smooth isometric embedding

$$
i: \quad H_{d+1} \longleftrightarrow \mathbb{R}^{d+2}: \quad x^{\mu} \longmapsto X^{A}\left(x^{\mu}\right) .
$$

From the above we can see that $\mathrm{AdS}_{d+1}$ space is homogeneous and isotropic, with isometry group $S O(d, 2)$. The corresponding algebra consists of the $\frac{1}{2}(d+1)(d+2)$ generators

$$
i J_{A B}=-i J_{B A}=\left(X_{A} \frac{\partial}{\partial X^{B}}-X_{B} \frac{\partial}{\partial X^{A}}\right),
$$

which satisfy the commutation relations

$$
\left[J_{A B}, J_{C D}\right]=i\left(\eta_{B C} J_{A D}+\eta_{A D} J_{B C}-\eta_{A C} J_{B D}-\eta_{B D} J_{A C}\right),
$$

with $\eta_{A B}=\operatorname{diag}(-++\cdots+-)$, known as 'conformal signature'. For calculations it is often convenient to work in Euclidean AdS, which can be reached by instead working in an ambient space Lorentzian signature $\eta_{A B}=\operatorname{diag}(-+\cdots+)$.

It is often convenient to use the following basis for the $\mathfrak{s o}(d, 2)$ generators (1.6),

$$
M_{i j}=i J_{i j}, \quad P_{i}^{ \pm}=J_{0 i} \pm i J_{i(d+1)}, \quad E=J_{0(d+1)},
$$

with commutators (all others are vanishing)

$$
\begin{gathered}
{\left[E, P_{i}^{ \pm}\right]= \pm P_{i}^{ \pm}, \quad\left[M_{i j}, P_{k}^{ \pm}\right]=\delta_{k j} P_{i}^{ \pm}-\delta_{k i} P_{j}^{ \pm}} \\
{\left[M_{i j}, M_{k l}\right]=\delta_{i k} M_{j l}+\delta_{j l} M_{i k}-\delta_{j k} M_{i l}-\delta_{i l} M_{j k}, \quad\left[P_{i}^{+}, P_{j}^{-}\right]=2 M_{i j}-2 \delta_{i j} E}
\end{gathered}
$$

where $i, j=1, \ldots, d$.

\section{Euclidean AdS in Poincaré Co-ordinates}

For concreteness, the co-ordinate system we'll most often use is Euclidean AdS in Poincaré co-ordinates $x^{\mu}=\left(z, y^{i}\right)$. With this choice our hyperboloid (1.2) is parameterised by

$$
\begin{aligned}
X^{0}(x) & =R \frac{z^{2}+y^{2}+1}{2 z} \\
X^{d+1}(x) & =R \frac{1-z^{2}-y^{2}}{2 z} \\
X^{i}(x) & =\frac{R y^{i}}{z} .
\end{aligned}
$$

Pulling back the ambient metric $\eta_{A B}$ one recovers the AdS metric in Poincaré co-ordinates

$$
d s^{2}=\left(\frac{\partial X^{A}}{\partial x^{\mu}} \frac{\partial X^{B}}{\partial x^{v}} \eta_{A B}\right) d x^{\mu} d x^{v}=\frac{R^{2}}{z^{2}}\left(d z^{2}+\delta_{i j} d y^{i} d y^{j}\right)
$$


where we used the Lorentzian signature $\eta_{A B}=\operatorname{diag}(-+\cdots+)$.

The usual AdS Killing tensors can be obtained in Poincaré co-ordinates by noting that

$$
\eta^{A B} \frac{\partial}{\partial X^{B}}=g^{\mu \nu} \frac{\partial X^{A}}{\partial x^{v}} \frac{\partial}{\partial x^{\mu}}-\frac{X^{A}}{R^{2}} X \cdot \partial_{X}
$$

which gives

$$
\begin{aligned}
i J^{A B} & =g^{\mu v}\left(X^{A} \frac{\partial X^{B}}{\partial x^{v}}-X^{B} \frac{\partial X^{A}}{\partial x^{v}}\right) \frac{\partial}{\partial x^{\mu}} \\
& =\frac{z^{2}}{R^{2}}\left[\left(X^{A} \frac{\partial X^{B}}{\partial z}-X^{B} \frac{\partial X^{A}}{\partial z}\right) \frac{\partial}{\partial z}+\delta^{i j}\left(X^{A} \frac{\partial X^{B}}{\partial y^{j}}-X^{B} \frac{\partial X^{A}}{\partial y^{j}}\right) \frac{\partial}{\partial y^{i}}\right] .
\end{aligned}
$$

For example, one recovers

$$
i E=J_{0(d+1)}=z \partial_{z}+y \cdot \partial_{y} .
$$

\subsection{The Conformal Boundary}

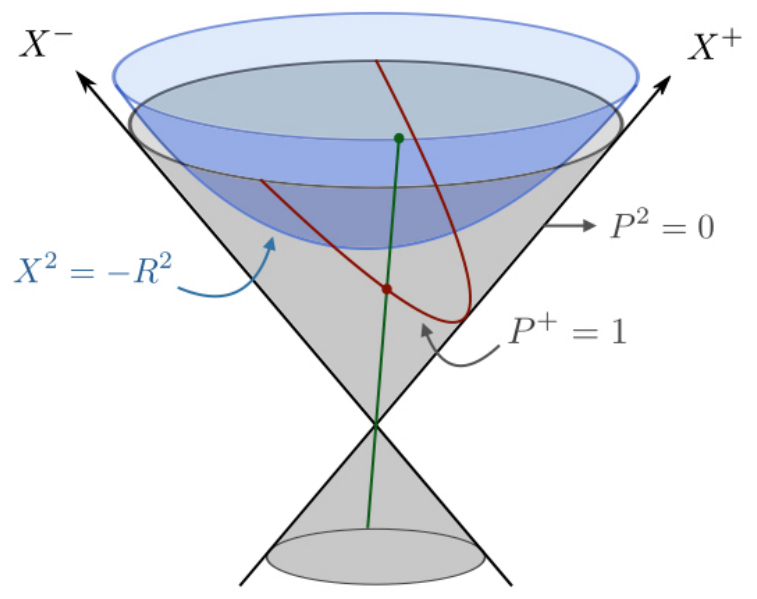

Figure 1:

Towards the $\mathrm{AdS}_{d+1}$ boundary, the hyperboloid (1.2) asymptotes to a light cone in the flat ambient space (see fig. 1). While this limit does not yield a well-defined boundary metric (see (1.13) for $z \rightarrow 0$ ), we can obtain a finite limit by considering the projective cone of light rays with co-ordinates

$$
P^{A} \equiv \varepsilon X^{A}, \quad \varepsilon \rightarrow 0
$$

Since $X^{2}$ is fixed, these null projective co-ordinates satisfy

$$
P^{2}=0, \quad P \sim \lambda P, \quad \lambda \neq 0,
$$


where in general $\lambda$ depends on $P$. The right-most statement tells us that, being rays, $P$ and $\lambda P$ are identified. This quotienting of the null cone identifies the $P$ with $d$-dimensional Minkowski space (with a point at infinity added) or any conformally flat manifold. ${ }^{4}$

In particular, the AdS boundary is parameterised by a Poincaré section of the null cone: $P^{+}=$ $P^{d+1}+P^{0}=$ constant. With the gauge choice $P^{+}=1$, we have

$$
P^{0}(y)=\frac{1}{2}\left(1+y^{2}\right), \quad P^{d+1}(y)=\frac{1}{2}\left(1-y^{2}\right), \quad P^{i}(y)=y^{i} .
$$

This is illustrated in fig. 1. The $S O(d, 2)$ isometry of $\mathrm{AdS}$ acts on the co-ordinates $P$ as a group of conformal symmetries, with a given transformation relating the section (1.19) to others with $d P^{+}=0$.

The usual conformal generators on the boundary are recovered from

$$
i J_{A B}=-i J_{B A}=\left(P_{A} \frac{\partial}{\partial P^{B}}-P_{B} \frac{\partial}{\partial P^{A}}\right),
$$

and identifying the combinations (1.7). Like for the AdS Killing tensors in the previous section $\S 1.1$, one can use that

$$
\begin{aligned}
\eta^{A B} \frac{\partial}{\partial P^{B}} & =\left(\frac{\partial P^{A}}{\partial y^{i}} \frac{\partial P^{B}}{\partial y^{i}}-Q^{A} P^{B}-Q^{B} P^{A}\right) \frac{\partial}{\partial P^{B}} \\
& =\delta^{i j} \frac{\partial P^{A}}{\partial y^{i}} \frac{\partial}{\partial y^{j}}-Q^{A} P \cdot \partial_{P}-P^{A} Q \cdot \partial_{P}
\end{aligned}
$$

where we employed (3.17) with $Q^{A}=(1, \mathbf{0},-1)$. This gives

$$
i J^{A B}=\left(P^{A} \frac{\partial P^{B}}{\partial y^{i}}-P^{B} \frac{\partial P^{A}}{\partial y^{i}}\right) \frac{\partial}{\partial y^{i}}+\left(P^{B} Q^{A}-P^{A} Q^{B}\right) y \cdot \partial_{y}
$$

where one notes that $P \cdot \partial_{P}=y \cdot \partial_{y}$. For example, for dilatations we recover

$$
i E=i J_{0(d+1)}=y \cdot \partial_{y} .
$$

\subsection{Unitary Irreducible Representations: Particles in AdS}

All possible types of elementary particles are defined by unitary irreducible representations (UIRs) of the space-time isometry. For studying the UIRs of the AdS isometry, it is useful to employ the basis (1.9) for the $\mathfrak{s o}(d, 2)$ generators. Since we are interested in unitarity representations of $\mathfrak{s o}(d, 2)$, the required Hermiticity condition $J_{A B}^{\dagger}=J_{A B}$ translates into

$$
E^{\dagger}=E, \quad\left(P^{ \pm}\right)^{\dagger}=P^{\mp}, \quad M_{i j}^{\dagger}=-M_{i j} .
$$

Generators $E$ and $M_{i j}$ comprise the maximally compact subgroup, $S O(2) \times S O(d)$, of $S O(d, 2)$. $E$ gives rise to rotations in the purely time-like $\left(X_{0}, X_{d+1}\right)$ plane and therefore identified with the

\footnotetext{
${ }^{4}$ Note that these redundant rescalings by $\lambda(y)$ are equivalent to sending $\varepsilon \rightarrow \varepsilon / \lambda$, so $P \rightarrow \lambda(y) P$ rescales the metric by an overall factor - as per the definition of a conformal transformation. The redundancy can thus be used to determine the transformation properties of a function $f(P)$ under a dilatation. If $f$ has scaling dimension $\Delta$, then $f(\lambda P)=\lambda^{-\Delta} f(P)$.
} 
Hamiltonian for AdS physics. The $M_{i j}$ give rotations of $S^{d-1}$ and are angular momentum generators. The remaining non-compact generators $P^{ \pm}$raise and lower the energy eigenvalue by one unit respectively, as can be seen from the $\left[E, J^{ \pm}\right]$commutator in (1.8).

UIRs of the AdS isometry group are thus labelled by the energy eigenvalue $\Delta$ and $\operatorname{spin} \underline{s}$ of its ground state $|\Delta, \underline{s}\rangle$

$$
E|\Delta, \underline{s}\rangle=\Delta|\Delta, \underline{s}\rangle, \quad P^{-}|\Delta, \underline{s}\rangle=0,
$$

which forms a unitary module of the $\mathfrak{s o}(2) \oplus \mathfrak{s o}(d)$ maximally compact sub-algebra. The spin $\underline{s}$ characterises the $\mathfrak{s o}(d)$ module, which is generically given by a collection of positive integers $\underline{s}=\left(s_{1}, \ldots, s_{r}\right)$ corresponding to the $\mathfrak{s o}(d)$ Young diagram

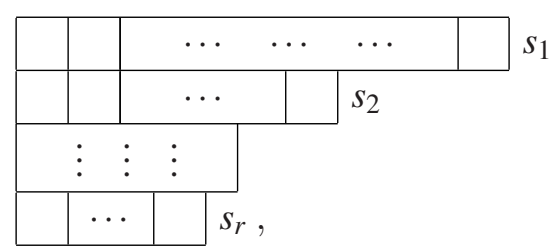

with $s_{1} \geq s_{2} \ldots \geq s_{r}$. For the purpose of these lectures (and from this point onwards) we only consider totally symmetric spin- $s$ representations $V_{s}$, which concern only the single row Young diagrams $\underline{s}=(s, 0, \ldots, 0)$,

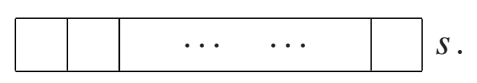

Given the ground state (1.24) of our spin-s particle, we can construct excited states furnishing the representation Fock space by applying the raising operator $P^{+}$. The complete $\mathfrak{s o}(d, 2)$ module $\mathscr{D}(\Delta, s)$ is therefore spanned by states of the schematic form ${ }^{5}$

$$
|\Delta, s\rangle_{n, l}=\left(P^{+} \cdot P^{+}\right)^{n} P_{i_{1}}^{+} \ldots P_{i_{l}}^{+}|\Delta, s\rangle, \quad n, l=0,1,2 \ldots,
$$

with energy eigenvalue $\Delta+2 n+l$. Note that $|\Delta, s\rangle_{0,0}=|\Delta, s\rangle$.

\section{Exercise 1.1: Quadratic Casimir}

Casimir operators of a given Lie algebra are distinguished operators which commute with each generator. Their eigenvalues thus characterise the irreducible representations, taking the same value for any state in a given representation.

The quadratic Casimir of the $\operatorname{AdS}_{d+1}$ isometry algebra is given by

$$
\mathscr{C}_{2}(\mathfrak{s o}(d, 2)) \equiv \frac{1}{2} J_{A B} J^{A B}=E(E-d)+\mathscr{C}_{2}(\mathfrak{s o}(d))-\delta^{i j} P_{i}^{+} P_{j}^{-} .
$$

Given that the $\mathfrak{s o}(d)$ Casimir $^{6} \mathscr{C}_{2}(\mathfrak{s o}(d))=-\frac{1}{2} M_{i j} M^{i j}$ has eigenvalue

$$
\left\langle\mathscr{C}_{2}(\mathfrak{s} o(d))\right\rangle=s(s+d-2),
$$

\footnotetext{
${ }^{5}$ In particular this is schematic for $l>0$, as, strictly speaking, the indices should be symmetrised in order to be irreducible $S O(d)$ tensors.
} 
on totally symmetric spin-s representations $V_{s}$, show that

$$
\left\langle\mathscr{C}_{2}(\mathfrak{s} o(d, 2))\right\rangle=\Delta(\Delta-d)+s(s+d-2),
$$

for states in the module $\mathscr{D}(\Delta, s)$.

Show also that

$$
\mathscr{C}_{2}(\mathfrak{s} o(d, 2))=R^{2} \square_{\mathrm{AdS}}+\mathscr{C}_{2}(\mathfrak{s} o(d, 1)),
$$

where $\square_{\mathrm{AdS}}=\nabla^{\mu} \nabla_{\mu}$ is the covariantised d'Alambertian operator in AdS and $\mathscr{C}_{2}(\mathfrak{s o}(d, 1))$ is the quadratic Lorentz Casimir in $(d+1)$-dimensions.

In the language of QFT, to our spin-s particle on AdS is associated a rank $s$ field $\varphi_{\mu_{1} \ldots \mu_{s}}$ which, as a carrier of $\mathscr{D}(\Delta, s)$, is totally symmetric and satisfies the Fierz-Pauli conditions

$$
\begin{gathered}
\left(\mathscr{C}_{2}(\mathfrak{s o} o(d, 2))-\left\langle\mathscr{C}_{2}(\mathfrak{s o}(d, 2))\right\rangle\right) \varphi_{\mu_{1} \ldots \mu_{s}}=0 \\
\nabla^{\mu_{1}} \varphi_{\mu_{1} \ldots \mu_{s}}=0 \\
g^{\mu_{1} \mu_{2}} \varphi_{\mu_{1} \ldots \mu_{s}}=0 .
\end{gathered}
$$

The final two conditions ensure that $\varphi_{\mu_{1} \ldots \mu_{s}}$ sits in $V_{s}$, the totally symmetric irreducible spin- $s$ representation of $S O(d)$. From the first condition (1.32a) one deduces the equation of motion (see exercise 1.1 above)

$$
\left(\nabla^{2}-m_{s}^{2}\right) \varphi_{\mu_{1} \ldots \mu_{s}}=0, \quad\left(m_{s} R\right)^{2}=\Delta(\Delta-d)-s .
$$

Recall that $R$ is the AdS radius.

\section{Unitarity Bounds and Higher Spin Gauge Fields}

Let us emphasise that representations are only unitary for a certain range of $\Delta$. Outside of this, negative norm states appear in the Hilbert space. Unitarity bounds on $\Delta$ can be obtained by demanding positive norm for every state in the multiplet, which we detail below.

It is sufficient to consider the norm of the first level descendants,

$$
\left(P_{i}^{+}|\Delta, s\rangle\right)^{\dagger} P_{j}^{+}|\Delta, s\rangle=\left\langle\Delta, s\left|P_{i}^{-} P_{j}^{+}\right| \Delta, s\right\rangle=2 \Delta \delta_{i j}-2 \Sigma_{i j},
$$

where we used the $\mathfrak{s} o(d, 2)$ commutator (1.9) and $M_{i j}|\Delta, s\rangle^{a}=\left(\sum_{i j}\right)^{a} b|\Delta, s\rangle^{b}$, with $a, b$ indices for the $S O(d)$ representation $V_{s}$ of $|\Delta, s\rangle$. For unitarity we require that (1.34) is positive definite, which implies

$$
\Delta \geq \max . \text { Eigenvalue }\left[\left(\Sigma_{i j}\right)_{b}^{a}\right] .
$$

The state $P_{i}^{+}|\Delta, s\rangle$ sits in the $V_{1} \otimes V_{s}$ representation of $S O(d)$, where $V_{1}$ is the vector representation. The trick is to write

$$
\left(\Sigma_{i j}\right)^{a}{ }_{b}=\frac{1}{2}\left(L^{k l}\right)_{i j}\left(\Sigma_{k l}\right)^{a}{ }_{b},
$$

\footnotetext{
${ }^{6}$ Notice here the minus sign, since the generators $M_{i j}$ are anti-Hermitian, differing from the usual Hermitian generators $J_{i j}$ by a factor of $i$.
} 
where $\left(L^{k l}\right)_{i j}=\delta_{i}^{k} \delta_{j}^{l}-\delta_{j}^{k} \delta_{i}^{l}$ is the spin generator in $V_{1}$. Then, regarding $L^{k l}$ and $\Sigma_{k l}$ as operators acting on $V_{1} \otimes V_{s},(1.36)$ becomes

$$
\begin{aligned}
L^{k l} \Sigma_{k l} & =\frac{1}{2}\left((L+\Sigma)^{2}-L^{2}-\Sigma^{2}\right) \\
& =-\left.\left\langle\mathscr{C}_{2}(\mathfrak{s} o(d))\right\rangle\right|_{V_{1} \otimes V_{s}}+\left.\left\langle\mathscr{C}_{2}(\mathfrak{s o} o(d))\right\rangle\right|_{V_{1}}+\left.\left\langle\mathscr{C}_{2}(\mathfrak{s} o(d))\right\rangle\right|_{V_{s}} .
\end{aligned}
$$

We see that the maximum Eigenvalue of $L \cdot \Sigma$ is dictated by the minimal quadratic $\mathfrak{s o}(d)$ Casimir in $V_{1} \otimes V_{s}$. By decomposing ${ }^{7}$

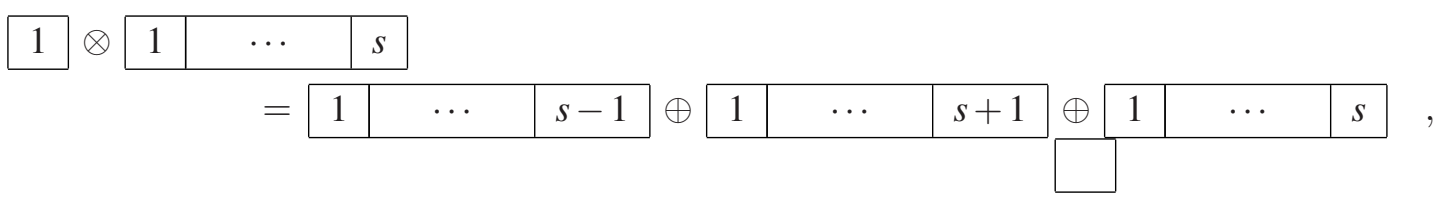

we see that this is given by $V_{s-1}$. We therefore obtain the bound

$$
\Delta \geq \frac{1}{2}\left(-\left.\left\langle\mathscr{C}_{2}(\mathfrak{s} o(d))\right\rangle\right|_{V_{s-1}}+\left.\left\langle\mathscr{C}_{2}(\mathfrak{s} o(d))\right\rangle\right|_{V_{1}}+\left.\left\langle\mathscr{C}_{2}(\mathfrak{s} o(d))\right\rangle\right|_{V_{s}}\right)=s+d-2 .
$$

While below this bound some states have negative norm, when it is saturated $(\Delta=s+d-2)$ null states emerge, which are orthogonal to all states in the Hilbert space. Such states hence form an invariant sub-module which should be quotiented out, corresponding to the emergence of a gauge symmetry. Indeed, one may verify the Fierz system (1.32) for $\Delta=s+d-2$ :

$$
\begin{gathered}
\left(R^{2} \nabla^{2}-(s+d-2)(s-2)+s\right) \varphi_{\mu_{1} \ldots \mu_{s}}=0 \\
\nabla^{\mu_{1}} \varphi_{\mu_{1} \ldots \mu_{s}}=0 \\
g^{\mu_{1} \mu_{2}} \varphi_{\mu_{1} \ldots \mu_{s}}=0
\end{gathered}
$$

is invariant under the gauge transformation

$$
\delta_{\xi} \varphi_{\mu_{1} \ldots \mu_{s}}=\nabla_{\left(\mu_{1}\right.} \xi_{\left.\mu_{2} \ldots \mu_{s}\right)}
$$

where the symmetric and traceless gauge parameter $\xi$ is on-shell:

$$
\begin{aligned}
\left(R^{2} \nabla^{2}-(s-1)(s+d-3)\right) \xi_{\mu_{1} \ldots \mu_{s-1}} & =0, \\
\nabla^{\mu_{1}} \xi_{\mu_{1} \ldots \mu_{s-1}} & =0, \\
g^{\mu_{1} \mu_{2}} \xi_{\mu_{1} \ldots \mu_{s-1}} & =0 .
\end{aligned}
$$

In contrast to our intuition from flat space, we see that gauge fields in AdS have a mass owing to the background curvature.

\footnotetext{
${ }^{7}$ Note that this decomposition holds only for $s>0$. For $s=0$ we have $V_{1} \otimes V_{s=0}=V_{1}$, leading to a modified bound, $\Delta \geq \frac{d}{2}-1$ or $\Delta=0$, for $s=0$. This gives the Breitenlohner-Freedman bound [40]

$$
m_{0}^{2}>-\left(\frac{d}{2 R}\right)^{2},
$$

which tells us that fields are still stable in AdS even if they are a little bit tachyonic.
} 


\section{Exercise 1.2: Generating Functions}

For manipulations of higher-rank tensors, it is useful to employ an operator notation. Fields are represented by generating functions, which for totally symmetric spin- $s$ representations read

$$
\varphi_{\mu_{1} \ldots \mu_{s}}(x) \longrightarrow \varphi_{s}(x, u)=\frac{1}{s !} \varphi_{\mu_{1} \ldots \mu_{s}}(x) u^{\mu_{1}} \ldots u^{\mu_{s}},
$$

where we have introduced the constant $(d+1)$-dimensional auxiliary vector $u^{\mu}$. The action of the covariant derivative also gets modified when acting on fields expressed as generating functions (1.46), owing to the viel-bein dependence

$$
\nabla_{\mu} \rightarrow \nabla_{\mu}+\omega_{\mu}^{a b} u_{a} \frac{\partial}{\partial u^{b}}, \quad\left[\nabla_{\mu}, \nabla_{v}\right]=\Lambda\left(u_{\mu} \partial_{u_{v}}-u_{v} \partial_{u_{\mu}}\right)+R_{\mu v \rho \sigma}^{\Lambda}(x) u^{\rho} \partial_{u_{\sigma}} .
$$

$\omega_{\mu}^{a b}$ is the spin-connection and viel-bein $e_{\mu}^{a}(x)$, with $u^{a}=e_{\mu}^{a}(x) u^{\mu}$. As a consequence of the vielbein postulate, we have

$$
\left[\nabla_{\mu}, u^{v}\right]=0, \quad\left[\partial_{u^{\mu}}, \nabla_{v}\right]=0 .
$$

$R_{\mu \nu \rho \sigma}^{\Lambda}$ is the Riemann tensor minus its constant trace part:

$$
R_{\mu v \rho \sigma}^{\Lambda}=R_{\mu v \rho \sigma}-\Lambda\left(g_{\mu \rho} g_{v \sigma}-g_{v \rho} g_{\mu \sigma}\right) .
$$

In this framework, tensor operations are translated into an operator calculus, which simplifies manipulations significantly. The operations: box, divergence, symmetrised gradient, divergence, trace, symmetrised metric, and spin can be represented by the following operators:

$$
\text { box: }\left(\nabla \cdot \partial_{u}\right)(\nabla \cdot u), \quad \text { divergence: } \nabla \cdot \partial_{u}, \quad \text { sym. metric: } u^{2} \text {, }
$$

$$
\text { sym. gradient: } u \cdot \nabla, \quad \text { trace: } \partial_{u}^{2}, \quad \text { spin: } u \cdot \partial_{u} \text {. }
$$

As an exercise, reformulate the Fierz-system (1.32) in the language of generating functions. The linearised gauge transformation (1.42) takes the form

$$
\delta_{\xi} \varphi(x, u)=u \cdot \nabla \xi_{s-1}(x, u), \quad \xi_{s-1}(x, u)=\frac{1}{(s-1) !} \xi_{\mu_{1} \ldots \mu_{s-1}} u^{\mu_{1}} \ldots u^{\mu_{s-1}} .
$$

Using the commutators

$$
\begin{aligned}
{[\square, u \cdot \nabla]=} & \Lambda\left[u \cdot \nabla\left(2 u \cdot \partial_{u}+d-1\right)-2 u^{2} \nabla \cdot \partial_{u}\right] \\
& +2 R_{\mu v \rho \sigma}^{\Lambda} \nabla^{\mu} u^{v} u^{\rho} \partial_{u_{\sigma}}-\left(\nabla_{\sigma} R_{v \rho}^{\Lambda}-\nabla_{\rho} R_{v \sigma}^{\Lambda}\right) u^{v} u^{\rho} \partial_{u^{\sigma}}+R_{v \rho}^{\Lambda} u^{v} \nabla^{\rho} \\
{\left[\nabla \cdot \partial_{u}, u \cdot \nabla\right] } & =\square+\Lambda\left[u \cdot \partial_{u}\left(u \cdot \partial_{u}+d-2\right)-u^{2} \partial_{u}^{2}\right]+R_{\mu v \rho \sigma}^{\Lambda} u^{v} u^{\rho} \partial_{u_{\mu}} \partial_{u_{\sigma}}+R_{\mu v}^{\Lambda} u^{\mu} \partial_{u_{v}}
\end{aligned}
$$

show that invariance of (1.32b) under (1.51) implies (1.43). Show that invariance of (1.33) fixes the mass of a spin-s gauge field in AdS to be $\left(m_{s} R\right)^{2}=(s-2)(s+d-2)-s$.

Hint: For AdS backgrounds $R_{\mu v \rho \sigma}^{\Lambda}=C_{\mu v \rho \sigma}=0$. 


\subsection{Lagrangian Formulation}

As a starting point in the quest for constructing interactions in a possible non-linear higherspin action, we introduce the Lagrangian formulation of the free equations of motion (1.41) for a bosonic spin-s gauge field on $\operatorname{AdS}_{d+1}$. This description was obtained by Fronsdal in 1978 [6] (and together with Fang for half integer spin [7]). We do not delve far into the free Fronsdal formulation, ${ }^{8}$ only briefly reviewing here the pertinent details.

The first step is to take the Fierz system ((1.41) and (1.43)) off-shell, while keeping the correct number of physical degrees of freedom to describe a $\mathscr{D}(s+d-2, s)$ module. Towards deriving the complete on-shell system from a single equation, one deforms the Klein-Gordon equation (1.41a) with divergence and trace terms

$$
\left[\square-m_{s}^{2}+\alpha_{1}(u, \nabla)\left(\nabla \cdot \partial_{u}\right)+\alpha_{2}(u, \nabla)\left(\partial_{u} \cdot \partial_{u}\right)\right] \varphi_{s}(x, u)=0,
$$

to account for the divergence (1.41b) and trace (1.41c) conditions. The guiding principle to determine the differential operators $\alpha_{i}(u, \nabla)$ is gauge invariance: Demanding that the deformations are at most two-derivative fixes

$$
\begin{aligned}
& \alpha_{1}(u, \nabla)=-(u \cdot \nabla)\left(\nabla \cdot \partial_{u}\right), \\
& \alpha_{2}(u, \nabla)=-u^{2}+\frac{1}{2}(u \cdot \nabla)^{2},
\end{aligned}
$$

but with the additional proviso that the gauge parameter is traceless,

$$
\delta_{\xi} \varphi_{s}(x, u)=u \cdot \nabla \xi_{s-1}(x, u), \quad\left(\partial_{u} \cdot \partial_{u}\right) \xi_{s-1}(x, u)=0 .
$$

This also leads to a constraint on the field $\varphi_{s}$ : Its double-trace is invariant under the gauge transformation (1.56) and so by unitarity must be set to zero, $\left(\partial_{u} \cdot \partial_{u}\right)^{2} \varphi_{s}(x, u)=0$.

One may verify that the Fronsdal formulation carries the correct number of physical degrees of freedom to describe a spin-s gauge field, reducing to the Fierz system upon gauge fixing - see e.g. [43] for details.

The algebraic trace constraints in the Fronsdal formulation

$$
\left(\partial_{u} \cdot \partial_{u}\right)^{2} \varphi_{s}(x, u)=0, \quad\left(\partial_{u} \cdot \partial_{u}\right) \xi_{s-1}(x, u)=0,
$$

may seem unappealing, but they non-the-less achieve the goal of removing the derivative constraints, taking the Fierz system (1.41) off-shell. Forgoing the constraints (1.57) simply shifts the unconventional features elsewhere, such as: into additional auxiliary fields [44-46] and introducing non-localities $[47,48]$. In these lectures we stick with the Fronsdal formulation.

The equation of motion (1.53) can be written in the form

$$
\mathscr{F}_{s}\left(x, u, \nabla, \partial_{u}\right) \varphi_{s}(x, u)=0,
$$

where $\mathscr{F}_{s}$ is the so-called Fronsdal operator

$$
\mathscr{F}_{s}\left(x, u, \nabla, \partial_{u}\right)=\square-m_{s}^{2}-u^{2}\left(\partial_{u} \cdot \partial_{u}\right)-(u \cdot \nabla)\left(\left(\nabla \cdot \partial_{u}\right)-\frac{1}{2}(u \cdot \nabla)\left(\partial_{u} \cdot \partial_{u}\right)\right) .
$$

\footnotetext{
${ }^{8}$ See, for instance, the reviews [41-43].
} 
This can be derived from the free action

$$
S_{\mathrm{AdS}}^{(2)}\left[\varphi_{s}\right]=\frac{s !}{2} \int_{\operatorname{AdS}_{d+1}} \varphi_{s}\left(x ; \partial_{u}\right) \mathscr{G}_{S}(x ; u),
$$

where $\mathscr{G}_{s}$ generalises to spin- $s$ gauge fields the linearised Einstein tensor

$$
\mathscr{G}_{S}(x ; u)=\left(1-\frac{1}{4} u^{2} \partial_{u} \cdot \partial_{u}\right) \mathscr{F}_{s}\left(x ; u, \nabla, \partial_{u}\right) \varphi_{s}(x, u) .
$$

Note that, crucially, the double-traceless condition on $\varphi_{s}$ ensures that the Bianchi identity is satisfied

$$
\left(\partial_{u} \cdot \nabla\right) \mathscr{G}_{S}(x, u)=0 .
$$

With the free action of a totally symmetric spin- $s$ gauge field on $\mathrm{AdS}_{d+1}$, naturally the next step is to ask if we can construct interactions. Like for the determination of the kinetic term (1.60), this search is underpinned by the requirement of gauge invariance, and has been subject to decades of intense efforts. So far this approach has led to results for all possible cubic interactions [49-69] that may appear in a non-linear higher-spin action.

In the following section we introduce a recent alternative approach that emerged to studying higher-spin interactions, which employs the AdS/CFT correspondence. As we shall see, holography seems to naturally imply the existence of interacting higher-spin theories on an AdS background, and has the potential to push further the successes of more conventional methods mentioned in the previous paragraph.

\section{The AdS/CFT Correspondence and Higher Spins}

In its most general form, the AdS / CFT correspondence [25-27] is a conjectured duality which can be elegantly formulated as a simple equation:

$$
\mathrm{AdS}_{d+1} \mathrm{QG} \stackrel{?}{=} \mathrm{CFT}_{d}
$$

In words: Quantum Gravity ${ }^{9}$ in asymptotically anti-de Sitter spacetime $\operatorname{AdS}_{d+1}$ is postulated to be equal to a non-gravitational conformal field theory (CFT). This is known as a holographic duality, since the CFT lives in (at least) one lower dimension. Since the boundary of asymptotically AdS spaces are conformally flat, we can regard the $\mathrm{CFT}_{d}$ as living on the 'conformal boundary' of the dual theory in $\operatorname{AdS}_{d+1}$. This is often depicted as in fig. 2.

In these lectures we are interested in a particular limit of the statement (2.1), in which higherspin gauge fields are present in $\mathrm{AdS}_{d+1}$. We thus won't delve into the details of this remarkable duality here, ${ }^{10}$ covering only the salient concepts.

The equivalence (2.1) is striking as, if true, it opens up the possibility to study gravity theories from the perspective of their CFT duals, and vice versa. Typically the dimensionless coupling $\lambda$

\footnotetext{
${ }^{9}$ To be a bit more cautious we could say: Any theory that we know how to define in the UV and behaves as ordinary gravity plus QFT in the infrared.

${ }^{10}$ Reviews of this vast topic include: [70-74].
} 


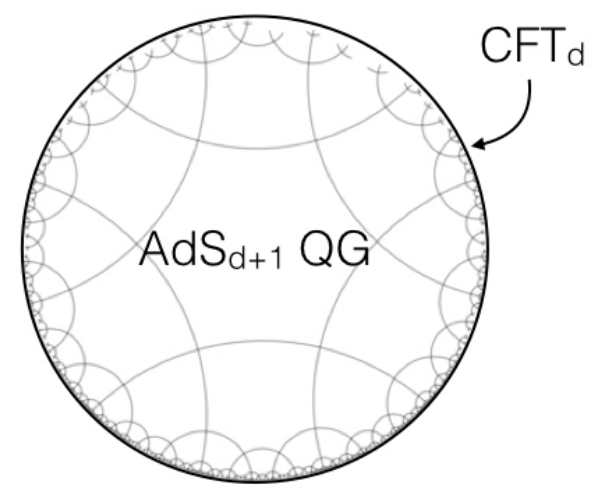

Figure 2: For a given dual pair (2.1), we can place the $\mathrm{CFT}_{d}$ on the boundary (solid black boundary of disc) asymptotically AdS boundary of the dual gravity theory (entire disc). The grey curves are geodesics. This perspective can be obtained by taking Euclidean AdS, adding a point at infinity to the $\mathbb{R}^{d}$ boundary and compactifying it to $S^{d}$.

of the $\mathrm{CFT}_{d}$ is related to the scale $\sqrt{\alpha^{\prime}}$ at which our gravity theory is sensitive to higher-derivative corrections via

$$
\lambda \sim\left(\frac{R^{2}}{\alpha^{\prime}}\right)^{d / 2} .
$$

In a string theory context we have $\alpha^{\prime}=l_{s}^{2}$, the square of the string length. From the relationship (2.2) we see that the holographic duality (2.1) is strong-weak in nature, with two interesting limits:

1. The point-particle limit: $\alpha^{\prime} / R^{2} \rightarrow 0$, where the CFT coupling grows large $\lambda>>1$.

2. The high energy limit: $\alpha^{\prime} / R^{2} \rightarrow \infty$, in which $\lambda \rightarrow 0$.

Although 1. has been subject to intense study to mine the possibilities of investigating strongly coupled systems via relatively well understood General Relativity, ${ }^{11}$ 2. underpins the topic of these lectures.

Why should the limit $\alpha^{\prime} / R^{2} \rightarrow \infty$ be interesting? In this regime it is intuited that an infinitedimensional symmetry may emerge, responsible for the good high energy behaviour of a UVcomplete theory of gravity: ${ }^{12}$ To understand this expectation in more concrete terms, consider one of the most promising candidates for a complete theory of gravity: String Theory. Taking simply the open bosonic string in flat space, for the states on the first Regge trajectory, we have

$$
\alpha^{\prime} m_{s}^{2}=s-1, \quad s=0,1,2,3, \ldots
$$

In the $\alpha^{\prime} \rightarrow \infty$ limit we indeed recover a tower of higher-spin gauge fields in the spectrum, whose non-trivial interactions would generate an infinite-dimensional, higher-spin symmetry [24]. This phenomena can also be observed by considering higher-derivative counter-terms added to the Einstein-Hilbert action [22].

\footnotetext{
${ }^{11}$ See $[75,76]$ for pedagogical introductions.

${ }^{12} \mathrm{~A}$ well known example of this phenomenon is given by the Standard model of electro-weak interactions, where the massive $W^{ \pm}$and $Z$ bosons arise from the spontaneous breaking of an $S U(2) \times U(1)$ symmetry, which emerges at high energies.
} 
With a lot of symmetry comes a lot of control. In this regard, uncovering an infinite-dimensional higher-spin symmetry principle could shed some light on the elusive high-energy behaviour of gravity. This makes the limit $\mathbf{2}$. even more profound, since, via holography, this highly symmetric phase of gravity can be probed through very simple, solvable, CFTs. In fact the emergence of higher-spin symmetry can also be seen from the dual CFT perspective: As we shall illustrate later, owing to the presence of a tower of conserved currents unbounded in spin in their spectrum, free CFTs are governed by an infinite-dimensional higher-spin symmetry. For the duality to hold, the theory in AdS should be governed by the same symmetry, making the existence of a highly symmetric phase of gravity even more plausible and more tractable to study.

In order to study this regime of holography in more detail, in the following we make the dictionary between the bulk and boundary theories more precise.

\subsection{The GKP/W Formula}

In practice it is most convenient to formulate the holographic duality (2.1) in terms of generating functions, in Euclidean signature. For concreteness, we work in Poincaré co-ordinates (1.13).

Let's start with the CFT side of the story. The generating function $F_{\mathrm{CFT}}[\bar{\varphi}]$ of connected correlators in a CFT admits a path-integral representation,

$$
\exp \left(-F_{\mathrm{CFT}}[\bar{\varphi}]\right)=\int D \phi \exp \left(-S_{\mathrm{CFT}}[\phi]+\int d^{d} y \bar{\varphi}(y) \mathscr{O}(y)\right) .
$$

We use $\phi$ to collectively denote the fundamental field(s) in the theory, governed by the CFT action $S_{\mathrm{CFT}}[\phi]$. The operator $\mathscr{O}$ is built from the fields $\phi$ in a gauge-invariant manner, and is sourced by $\bar{\varphi}(y)$. The source is not dynamical, rather a function that is fixed and under our control.

Holography breathes life into the source $\bar{\varphi}$ of our CFT operators: Regarding the $\mathrm{CFT}_{d}$ as living on the boundary of $\mathrm{AdS}_{d+1}$ (like in fig. 2), the source is promoted to a fully fledged dynamical field $\varphi(y, z)$ in AdS governed by the bulk action $S_{\mathrm{AdS}}[\varphi]$. The only control we have over $\varphi$ is its boundary value $\bar{\varphi}(y)$ at $z=0$.

The GKP/W formula $[26,27,77]$ puts the above holographic picture on a more concrete footing. It states that the physical quantity $F_{\mathrm{CFT}}[\bar{\varphi}]$ in the CFT coincides with the AdS one $\Gamma_{\mathrm{AdS}}[\bar{\varphi}]$,

$$
F_{\mathrm{CFT}}[\bar{\varphi}]=\Gamma_{\mathrm{AdS}}[\bar{\varphi}]
$$

with

$$
\exp \left(-\Gamma_{\mathrm{AdS}}[\bar{\varphi}]\right)=\int_{\left.\varphi\right|_{\partial \mathrm{AdS}}=\bar{\varphi}} \mathscr{D} \varphi \exp \left(-\frac{1}{G} S_{\mathrm{AdS}}[\varphi]\right)
$$

the bulk partition function and $G$ the gravitational constant. This identification and the precise boundary conditions are made more precise in the following section.

The GKP/W formula provides a prescription for computing correlation functions of gauge invariant operators in the CFT using the dual gravity theory. Connected correlation functions for instance can be obtained by functionally differentiating $\Gamma_{\text {AdS }}[\bar{\varphi}]$ instead:

$$
\left\langle\mathscr{O}_{1}\left(y_{1}\right) \ldots \mathscr{O}_{n}\left(y_{n}\right)\right\rangle_{\mathrm{conn} .}=\left.(-1)^{n} \frac{\delta}{\delta \bar{\varphi}_{1}\left(y_{1}\right)} \ldots \frac{\delta}{\delta \bar{\varphi}_{n}\left(y_{n}\right)} \Gamma_{\mathrm{AdS}}\left[\bar{\varphi}_{i}\right]\right|_{\bar{\varphi}_{i}=0} .
$$


In this holographic picture each functional derivative fires a $\varphi_{i}(y, z)$ particle into AdS.

At first sight the GKP/W formula doesn't appear to be so useful, since it involves dealing with the full gravity partition function. However, we may always take the limit in which gravity is classical. But what does this mean for the CFT? It turns out that quantum corrections in the bulk are sensitive to the number of degrees of freedom $N_{\text {dof. }}$ in the CFT, with ${ }^{13}$

$$
\left(\frac{R}{\ell_{p}}\right)^{d-1} \sim N_{\text {dof. }}
$$

The classical limit in the bulk thus corresponds to having a large number of degrees of freedom in the dual CFT, $N_{\text {dof }}>>1$. In particular, the weak coupling $G<<R$ expansion

$$
\Gamma_{\text {AdS }}[\bar{\varphi}]=\frac{1}{G} \Gamma_{\text {AdS }}[\bar{\varphi}]^{(0)}+\Gamma_{\text {AdS }}[\bar{\varphi}]^{(1)}+G \Gamma_{\text {AdS }}[\bar{\varphi}]^{(2)}+\ldots,
$$

which is regulated by the dimensionless coupling

$$
g:=R^{1-d} G \sim 1 / N_{\mathrm{dof}}+\mathscr{O}\left(1 / N_{\mathrm{dof}}^{2}\right),
$$

translates to a large $N_{\text {dof }}$ expansion in the CFT,

$$
F_{\mathrm{CFT}}=N_{\mathrm{dof}} F_{\mathrm{CFT}}^{(0)}+F_{\mathrm{CFT}}^{(1)}+\frac{1}{N_{\mathrm{dof}}} F_{\mathrm{CFT}}^{(2)}+\ldots
$$

Given that the expansion (2.10) encodes the one-particle irreducible scattering amplitudes of the $\varphi_{i}$ in AdS, we encounter an elegant diagrammatic holographic interpretation of connected CFT correlators at large $N_{\text {dof }}$ :

$$
\left\langle\mathcal{O}_{1}\left(y_{1}\right) \ldots \mathcal{O}_{n}\left(y_{n}\right)\right\rangle_{\text {conn. }}=\sum_{k=0}^{\infty} G^{k-1}
$$

The bulk diagrams on the right hand side are known as Witten diagrams. They were first computed in the context of the limit 1. of the duality in [78-99]. More recently Witten diagrams in the context of the limit 2. where considered in [32-35, 100-106]. One-loop vacuum energies ( $n=0$ and $k=1$ in (2.13)) have been computed in [107-116].

\footnotetext{
${ }^{13} \mathrm{We}$ can arrive to this by recalling that degrees of freedom in a CFT can be roughly measured by the overall coefficient $\mathrm{C}_{T}$ of the energy momentum tensor two-point function. From the GKP/W formula, we identify

$$
\langle T T\rangle_{\mathrm{CFT}} \sim \mathrm{C}_{T} \sim N_{\text {dof. }} \quad \leftrightarrow \quad\langle g g\rangle_{\mathrm{AdS}} \sim \frac{R^{d-1}}{G} \sim\left(\frac{R}{\ell_{p}}\right)^{d-1},
$$

since, as we shall see explicitly in the following section, the CFT energy momentum tensor is dual to the graviton in AdS.
} 


\section{The Field-Operator Map}

What does it take for a field $\varphi$ in an asymptotically AdS space to source an operator $\mathscr{O}$ of energy $\Delta$ and spin-s in the dual CFT?

1. The CFT operator must be gauge invariant, since the bulk physics is not sensitive to gauge dependent quantities $\rightarrow$ Spectrum of local operators are composed of traces. ${ }^{14}$

2. The boundary value of $\varphi$ should have the same transformation properties under $S O(d, 2)$ (quantum numbers) as the source to $\mathscr{O}$.

How do we ensure condition 2? As we saw in $\S 1.3$, the quantum numbers of $S O(d, 2)$ are energy and spin. By requiring invariance of (2.4) under dilatations, the source $\bar{\varphi}$ of $\mathscr{O}$ has energy $\tilde{\Delta}=d-\Delta$. We therefore have ${ }^{15}$

$$
[E, \bar{\varphi}(y)]=-i(d-\Delta+y \cdot \partial) \bar{\varphi}(y),
$$

while on $\varphi$ it acts via a Lie derivative

$$
\mathscr{L}_{E} \varphi^{\mu_{1} \ldots \mu_{s}}(z, y)=-i\left(z \partial_{z}+y \cdot \partial_{y}-s\right) \varphi^{\mu_{1} \ldots \mu_{s}}(z, y) .
$$

By comparing (2.15) with (2.14), to preserve invariance under conformal transformations as $z \rightarrow 0$ we need, for boundary directions $i$,

$$
\varphi^{i_{1} \ldots i_{s}} \sim z^{d+s-\Delta} \quad \text { as } \quad z \rightarrow 0,
$$

where the coefficient $\varphi$ of $z^{d+s-\Delta}$ gives the source to $\mathscr{O}$.

A special case of the field-operator map occurs when the unitarity bound is saturated (i.e. when $\Delta=s+d-2$ ), which is of interest from our perspective of higher-spin holography. Recall from $\S 1.3$ that such representions of $S O(d, 2)$ are known as short representations owing to the appearence of zero norm states in the Fock space, which should be factored out. For the bulk field $\varphi$, this multiplet shortening corresponds to the gauge invariance

$$
\delta_{\xi} \varphi_{\mu_{1} \ldots \mu_{s}}=\nabla_{\left(\mu_{1}\right.} \xi_{\left.\mu_{2} \ldots \mu_{s}\right)}
$$

In the CFT, this shortening manifests itself in the conservation of the lowest weight (primary) operator in the conformal multiplet:

$$
\partial^{i_{1}} \mathscr{O}_{i_{1} \ldots i_{s}} \approx 0 .
$$

Indeed, invariance of under the gauge variations (2.17) corresponds to the conservation of $\mathscr{O}$ :

$$
0=\delta_{\xi} \int_{\partial \mathrm{AdS}} d^{d} y \bar{\varphi}^{i_{1} \ldots i_{s}} \mathscr{O}_{i_{1} \ldots i_{S}} \stackrel{\mathrm{IBP}}{=}-\int_{\partial \mathrm{AdS}} d^{d} y \xi^{i_{2} \ldots i_{s}} \partial^{i_{1}} \mathscr{O}_{i_{1} \ldots i_{s}} \Longrightarrow \partial^{i_{1}} \mathscr{O}_{i_{1} \ldots i_{s}} \approx 0 .
$$

In summary, the field-operator map tells us that:

\footnotetext{
${ }^{14}$ In partciular, in the large $N_{\text {d.o.f. }}$ limit single-trace operators are identified with bulk single-particle states, while multi-trace operators are identified with multi-particle states in the bulk.

${ }^{15} \mathrm{See}$, for instance, [117] for a derivation of the action of the dilation generator.
} 


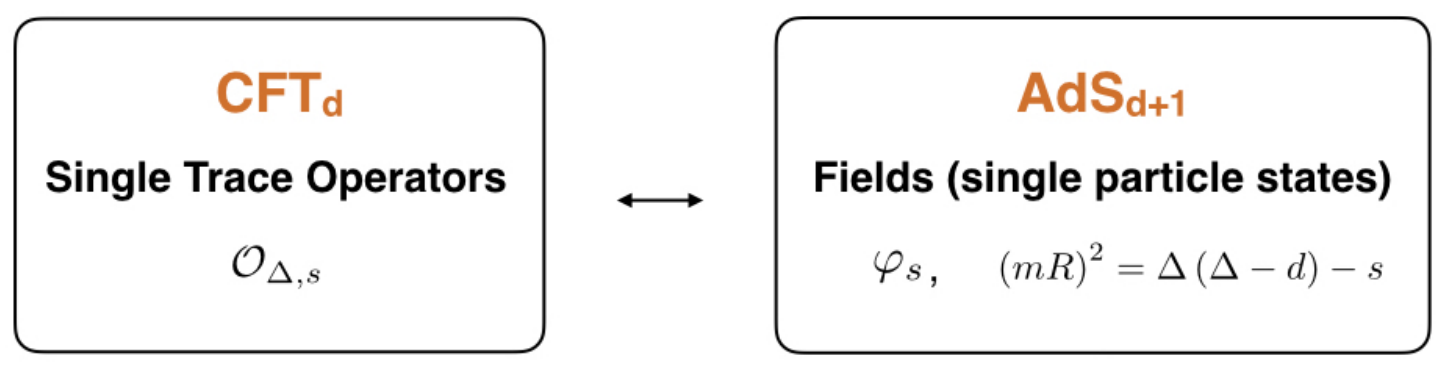

When $\Delta=s+d-2$ (saturation of unitarity bound):

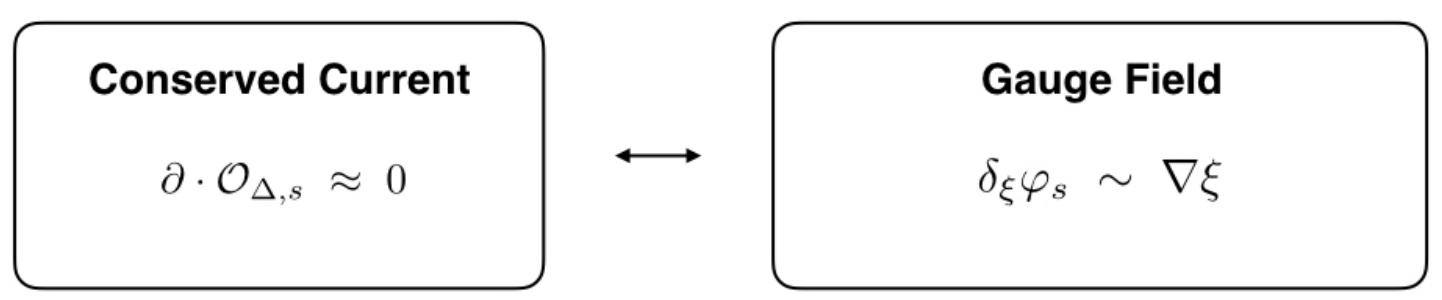

\subsection{Higher Spin Holography}

With the dictionary between CFT operators and bulk fields in place, let us explore in more detail holography in the limit 2. Recall that this is the regime in which the dual CFT becomes free.

For simplicity, consider a very basic example of a free CFT: A free massless $N$-component scalar in $d$-dimensions,

$$
S[\phi]=\frac{1}{2} \int d^{d} y \sum_{a=1}^{N} \partial_{i} \phi^{a} \partial^{i} \phi^{a}, \quad i=1, \ldots, d
$$

with equation of motion

$$
\partial^{2} \phi^{a}=0
$$

This is known as the free scalar $O(N)$ vector model. For a free scalar we have $\Delta=\frac{d}{2}-1$, and throughout we work in Euclidean signature.

In the $O(N)$ singlet sector (as relevant for holography), amongst the single-trace operators we have the conserved and traceless ${ }^{16}$ stress-energy tensor

$$
T_{i j}=\partial_{i} \phi^{a} \partial_{j} \phi^{a}-\frac{1}{2} \delta_{i j} \partial^{k} \phi^{a} \partial_{k} \phi^{a}-\frac{d-2}{4(d-1)}\left(\partial_{i} \partial_{j}-\delta_{i j} \partial^{2}\right) \phi^{a} \phi^{a}
$$

where the right-most term is an improvement which is separately conserved, which is added to make the stress-energy tensor traceless. While it is straightforward to verify conservation using the equation of motion (2.21), notice that the stress energy tensor has dimension $\Delta=d$, and thus saturates the unitarity bound for a spin-2 lowest weight operator.

It is well known that the presence of a conserved and traceless stress-energy tensor is the signal of conformal invariance. But, free CFTs possess also much larger, higher-spin, symmetry. In this

\footnotetext{
${ }^{16} \mathrm{As}$ required for a CFT.
} 
context, a higher-spin symmetry is typically ${ }^{17}$ an infinite dimensional extension of the conformal algebra, generated by a tower of conserved charges that are unbounded in spin. ${ }^{18}$

That higher-spin charges are present in the free scalar $O(N)$ model can be seen explicitly by considering the generating function

$$
\mathscr{J}(y ; q)=\phi^{a}(y+i q) \phi^{a}(y-i q)=\sum_{s=0}^{\infty} \frac{1}{s !} \mathscr{J}_{i_{1} \ldots i_{s}}(y) q^{i_{1}} \ldots q^{i_{s}},
$$

which describes a tower of operators $\mathscr{J}_{i_{1} \ldots i_{s}}$ of ranks $s=0,2,4, \ldots$ It is straightforward to verify that (2.23) is conserved, $\partial_{y} \cdot \partial_{q} \mathscr{J}(y ; q) \approx 0$, which in turn implies ${ }^{19}$

$$
\partial^{i_{1}} \mathscr{J}_{i_{1} \ldots i_{s}} \approx 0, \quad s=2,4,6, \ldots
$$

The theory is thus, as a consequence of Noether's theorem, governed by the higher-spin symmetry generated by the charges associated to the conserved currents (2.24). Let us note that for $s=0$ we have instead the scalar singlet operator

$$
\mathscr{O}=\phi^{a} \phi^{a}
$$

of dimension $\Delta=2 \times\left(\frac{d}{2}-1\right)=d-2$. Together with the tower of conserved currents (2.24), this comprises the entire single-trace sector of the theory.

A would-be dual theory in AdS should also be governed by (an appropriately gauged form) of the same higher-spin symmetry. According to the field-operator map, we expect the single-particle spectrum on $\operatorname{AdS}_{d+1}$ to consist of a tower of gauge fields $\varphi_{s}$ for each even spin $s=2,4,6,8, \ldots$ corresponding to each of the conserved currents (2.24) in the CFT, and a parity even scalar $\varphi_{0}$ dual to the scalar single trace operator $\mathscr{O}=\phi^{a} \phi^{a}[30,31]$.

While the discussion above was just representation theory, let us note that since (as we shall see explicitly) the correlation functions of operators in the singlet sector are non-trivial, owing to the GKP/W formula \$2.1, we expect non-trivial interactions amongst the higher-spin gauge fields on $\mathrm{AdS}_{d+1}$. In this regard, the AdS/CFT correspondence appears to make the potential existence of consistent interacting theories of higher-spin gauge fields quite natural.

Exercise 3.1: Higher Spin Conserved Currents

Extract the explicit form of the spin-s conserved currents from the generating function (2.23)

$$
\mathscr{J}_{i_{1} \ldots i_{s}}=i^{s} \sum_{k=0}^{s}(-1)^{k}\left(\begin{array}{l}
s \\
k
\end{array}\right) \partial_{\left(i_{1} \ldots \partial_{i_{k}}\right.} \phi^{a} \partial_{i_{k+1}} \ldots \partial_{\left.i_{s}\right)} \phi^{a}
$$

Hint:

$$
\phi^{a}(y+i q) \phi^{a}(y-i q)=e^{i q \cdot \partial_{x}} \phi^{a}(x) e^{-i q \cdot \partial_{x}} \phi^{a}(x)
$$

\footnotetext{
${ }^{17}$ In certain dimensions higher-spin algebras have finitely many generators, such as $d=2$. However here we work in general $d$.

${ }^{18}$ In fact in $d \geq 3$, assuming the existence of exactly one stress tensor, the presence of currents with spin $s \geq 3$ in a CFT implies that the theory is free [118-122].

${ }^{19}$ Since the scalar $\phi^{a}$ is real, odd spin operators do not appear in (2.23). See exercise 3.1.
} 
Notice that the currents (2.26) are not traceless, but, just like for the stress-energy tensor is CFT, one can add improvements to make them traceless.

One direct way [123] to obtain the improved current is to consider the generating function ansatz

$$
\mathscr{J}_{s}(y ; z)=\left.f^{(s)}\left(z \cdot \partial_{y_{1}}, z \cdot \partial_{y_{2}}\right) \phi^{a}\left(y_{1}\right) \phi^{a}\left(y_{2}\right)\right|_{y_{1}, y_{2} \rightarrow y},
$$

with null auxiliary vectors $z^{2}=0$ enforcing tracelessness. By demanding that $\mathscr{J}_{s}$ is annihilated by the conformal boost

$$
P^{-} \mathscr{J}_{s}=0
$$

as per the definition of a primary (lowest weight) CFT operator, show that

$$
\left[\left(\frac{d}{2}-1+y \partial_{y}\right) \partial_{y}+\left(\frac{d}{2}-1+\bar{y} \partial_{y}\right) \partial_{\bar{y}}\right] f^{(s)}(y, \bar{y})=0 .
$$

The solution is given in terms of a Gegenbauer polynomial

$$
f^{(s)}(y, \bar{y})=(y+\bar{y})^{s} C_{s}^{\left(\frac{d-2}{2}\right)}\left(\frac{y-\bar{y}}{y+\bar{y}}\right) .
$$

Notice also that (2.26) is zero for odd spins $s$. Show that by considering instead a complex scalar,

$$
\mathscr{I}(y ; q)=\phi^{a}(y+i q) \phi^{* a}(y-i q),
$$

as in the $U(N)$ theory, one obtains a tower of conserved currents for each integer spin.

It turns out the spectrum of the conjectured dual bulk theory is precisely that of the so-called minimal bosonic Vasiliev higher-spin theory in $\operatorname{AdS}_{d+1}$ [124], which was in fact developed (in $\mathrm{AdS}_{4}$ ) before the emergence of AdS/CFT [125]. The theory is formulated at the level of the equations of motion, ${ }^{20}$ by introducing an infinite-dimensional auxiliary internal space. ${ }^{21}$ Comparably little is known about higher-spin interactions without the auxiliary fields and in the 'metric-like' formalism, ${ }^{22}$ which would extend the free Fronsdal action (1.60) to the interacting level. On the other-hand the GKP/W formula (2.5) seems to provide a possible implicit definition of a non-linear on-shell action. In these lectures we shall demonstrate how to use the GKP/W formula to extract metric-like higher-spin interactions on $\mathrm{AdS}_{d+1}$, free from auxiliary fields.

Let us emphasise that above we have considered the simplest instance of higher-spin holography, where in the bulk one has just a tower of gauge fields of increasing spin. ${ }^{23}$ One may even question whether holography holds in this more general scenario. On the other hand, Vasiliev's theory may be considered as a toy-model for studying the first Regge trajectory of string theory on $\operatorname{AdS}_{d+1}$ in the tensionless limit (which, as we have seen, indeed consists of a tower of higher-

\footnotetext{
${ }^{20}$ See [126-128] for proposals for an action, using the machinery of auxiliary fields.

${ }^{21}$ See [129-131] for reviews of the Vasiliev system.

${ }^{22}$ It is called 'metric-like' because the metric is generalised by the totally symmetric tensor $\varphi_{\mu_{1} \ldots \mu_{s}}$.

${ }^{23}$ This is in contrast to the stacks of Regge trajectories like in String Theory, which is mirrored in the dual CFT description with the basic fields being in the adjoint representation, as opposed to the fundamental representation considered here.
} 
spin gauge fields). In this scenario, one is free to study the consequences of higher-spin symmetry without the added complication of coupling higher-spin gauge fields to matter. Recent efforts have been dedicated, with the guidance of holography, to embed Vasiliev's higher-spin theory into tensionless string theory [132-144]. The matter fields belonging to subleading Regge trajectories into multiplets of the higher-spin symmetry generated by the first Regge trajectory.

The set-up considered here is the simplest amongst AdS higher-spin / vector model dualities. In particular, the spectrum of single-particle states includes only even spin gauge fields. There exists an extension of this theory to one whose spectrum contains a gauge field for each integer spin. The conjectured dual CFT description is the free $U(N)$ invariant theory of a complex scalar field $\phi^{a} .{ }^{24}$ Further instances of higher-spin vector model dualities have been postulated, including: The so-called type-B theory in $\mathrm{AdS}_{4}$ with a parity odd scalar in the bulk, Chan-Paton factors and supersymmetry [140,145-147]. For comprehensive reviews on the higher-spin / vector model dualities, see: $[148,149]$.

To move towards our goal of studying higher-spin interactions from a holographic perspective, in the following sections we introduce some useful tools for dealing with higher-spin fields in the metric-like formalism.

\section{The Ambient Space Formalism}

The ambient space formalism is an instrumental tool in handling spinning fields and operators in the context of AdS/CFT. Dating back to Dirac [39], the basic idea is that fields in $\mathrm{AdS}_{d+2}$, and respectively dual operators in the $\mathrm{CFT}_{d}$, may be represented by fields in a $(d+2)$-dimensional flat ambient space. This makes manifest the mutual bulk and boundary $S O(d, 2)$ symmetry. For metriclike calculations in anti-de Sitter space, an attractive feature of the ambient space formalism is that expressions intrinsic to the AdS manifold (e.g. involving non-commuting covariant derivatives) can be expressed in terms of simpler-flat space ones of the ambient space (e.g. commuting partial derivatives). Owing to these simplifying features, the ambient formalism has enjoyed a wide variety of applications in AdS, HS and conformal field theory. For a flavour, see for example: $[65,150$ 154].

We review the basic features of the ambient approach here, and refer the reader to $[65,155,156]$ for further details.

\subsection{Bulk Fields}

Our goal is to formulate totally symmetric spin- $s$ unitary representations of the AdS isometry in the language of ambient space. A key point to keep in mind is that, in representing a field on AdS by a tensor living in the higher-dimensional ambient space, one has to make sure that the number degrees of freedom is kept constant.

In the ambient formalism a given smooth rank-r field $t_{\mu_{1} \ldots \mu_{r}}(x)$ intrinsic to the $\operatorname{AdS}_{d+1}$ manifold is assigned a representative $T_{A_{1} \ldots A_{r}}(X)$ of the same rank, which lives in the flat $(d+2)$ dimensional ambient space and in the same representation of $S O(d, 2)$. Naturally, the pull back of

\footnotetext{
${ }^{24}$ See exercise 3.1 .
} 
$T$ onto the AdS must satisfy

$$
i^{*}: T_{A_{1} \ldots A_{r}}(X) \longmapsto t_{\mu_{1} \ldots \mu_{r}}(x)=\frac{\partial X^{A_{1}}(x)}{\partial x^{\mu_{1}}} \ldots \frac{\partial X^{A_{r}}(x)}{\partial x^{\mu_{r}}} T_{A_{1} \ldots A_{r}}(X(x)) .
$$

However, at this level the choice of representative $T$ is not unique. Indeed, the $S O(d, 2)$ generators (1.6) are interior to AdS, $\left[J_{A B}, X^{2}+R^{2}\right]=0$, and are not sensitive to the extension of $T$ away from the AdS manifold. More explicitly, there are two sources of ambiguity:

1. Addition of tensors with components perpendicular to the AdS manifold, which sit in the kernel of the pullback (3.1):

$$
X^{2}=-\left.R^{2} \Longrightarrow \frac{\partial X}{\partial x^{\mu}} \cdot X\right|_{X^{2}=-R^{2}}=0 .
$$

2. Dependence on the radial direction $\rho=\sqrt{-X^{2}} \cdot{ }^{25}$

A prescription to fix the above ambiguities was provided by Fronsdal [151] in the '70s. For ambiguities of type 1, we impose that $T$ is tangent to submanifolds of constant $\rho:^{26}$

$$
X^{A_{i}} T_{A_{1} \ldots A_{i} \ldots A_{r}}=0, \quad i=1, \ldots, r .
$$

For the radial dependence, a simple (and thus convenient) condition is to impose that on-shell $T$ is a harmonic function in the ambient space

$$
\partial_{X}^{2} T_{A_{1} \ldots A_{r}}=0 .
$$

In order for (see exercise 4.1) $T$ to carry the correct representation of $S O(d, 2)$, the harmonic condition (3.6) implies that it is homogeneous

$$
\left(X \cdot \partial_{X}-\mu\right) T_{A_{1} \ldots A_{r}}=0, \quad \text { i.e. } \quad T_{A_{1} \ldots A_{r}}(\lambda X)=\lambda^{-\mu} T_{A_{1} \ldots A_{r}}(X),
$$

where we may choose either $\mu=\Delta$ or $\mu=d-\Delta$.

The above discussion also extends to the covariant derivative. The ambient representative of the covariant derivative $\nabla_{\mu}$ corresponding to the Levi-Civita connection on $\operatorname{AdS}_{d+1}$ is simply given by

and acts via

$$
\nabla_{A}=\mathscr{P}_{A}^{B} \frac{\partial}{\partial X^{B}}
$$

$$
\nabla=\mathscr{P} \circ \partial \circ \mathscr{P} .
$$

The first projection ensures that we are acting tangent to the AdS manifold. For example,

$$
\nabla_{B} T_{A_{1} \ldots A_{s}}=\mathscr{P}_{A}^{C} \mathscr{P}_{A_{1}}^{C_{1}} \ldots \mathscr{P}_{A_{s}}^{C_{r}} \frac{\partial}{\partial X^{C}}(\mathscr{P} T)_{C_{1} \ldots C_{s}} .
$$

\footnotetext{
${ }^{25}$ In particular, we are free to multiply by $\rho / R$ where $\rho$ is the radial co-ordinate $\rho=\sqrt{-X^{2}}$, or add terms proportional to $X^{2}+R^{2}$.
}

${ }^{26}$ This condition can be imposed by hand, applying the projection operator

$$
\mathscr{P}_{A}^{B}=\delta_{A}^{B}-\frac{X_{A} X^{B}}{X^{2}}
$$

which acts on ambient tensors as

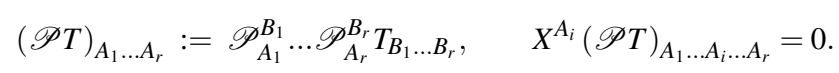




\subsection{Boundary Fields}

The above discussion also extends to fields living on the AdS boundary. In the same way, a rank-s field $f_{i_{1} \ldots i_{s}}(y)$ on the $\operatorname{AdS}_{d+1}$ boundary is represented in ambient space by a function $F_{A_{1} \ldots A_{s}}(P)$ living on a $P^{+}=$const. slice of the projective null cone $\S 1.2$.

If $f_{i_{1} \ldots i_{s}}$ is a symmetric spin-s boundary field of energy $\Delta$, its representative $F_{A_{1} \ldots A_{s}}(P)$ is also symmetric and satisfies

$$
\begin{aligned}
\eta^{A_{1} A_{2}} F_{A_{1} \ldots A_{s}}(P) & =0 \\
F_{A_{1} \ldots A_{s}}(\lambda P) & =\lambda^{-\Delta} F_{A_{1} \ldots A_{s}}(P), \quad \lambda(y)>0 .
\end{aligned}
$$

Like for the ambient description of bulk fields in the previous section, we impose that $F_{A_{1} \ldots A_{s}}(P)$ is transverse to the light-cone

$$
P^{A_{1}} F_{A_{1} \ldots A_{s}}(P)=0
$$

However in this case, since $P^{2}=0$, there is an extra redundancy

$$
\begin{gathered}
F_{A_{1} \ldots A_{s}}(P) \rightarrow F_{A_{1} \ldots A_{s}}(P)+P_{\left(A_{1}\right.} \Lambda_{\left.A_{2} \ldots A_{s}\right)} \\
P^{A_{1}} \Lambda_{A_{1} \ldots A_{s-1}}=0, \quad \Lambda_{A_{1} \ldots A_{s-1}}(\lambda P)=\lambda^{-(\Delta+1)} \Lambda_{A_{1} \ldots A_{s-1}}(P), \quad \eta^{A_{1} A_{2}} \Lambda_{A_{1} \ldots A_{s-1}}=0,
\end{gathered}
$$

which, together with (3.13), removes the extra two degrees of freedom per index of $F_{A_{1} \ldots A_{s}}$.

The scaling behaviour (3.12) extends the definition of $F_{A_{1} \ldots A_{s}}$ away from the $P^{+}=$const. slice, with the homogeneity degree fixed by the fact that $P \rightarrow \lambda(y) P$ re-scales the metric on $P^{+}=$const. by an overall factor - i.e. it implements a conformal transformation. See $\S 1.2$. The tracelessness condition (3.11) follows from the tracelessness of $f_{i_{1} \ldots i_{s}}$ : We have

$$
f_{i_{1} \ldots i_{r}}(y)=\frac{\partial P^{A_{1}}(y)}{\partial y^{i_{1}}} \ldots \frac{\partial P^{A_{r}}(y)}{\partial y^{i_{r}}} F_{A_{1} \ldots A_{r}}(P(y)) .
$$

In taking the trace of $f_{i_{1} \ldots i_{r}}(y)$, on the RHS we implement the contraction

$$
\delta^{i j} \frac{\partial P^{A}}{\partial y^{i}} \frac{\partial P^{B}}{\partial y^{j}}=\eta^{A B}+P^{A} Q^{B}+P^{B} Q^{A}, \quad \text { where } \quad Q^{A}=(1, \mathbf{0},-1) .
$$

This gives vanishing trace of $f_{i_{1} \ldots i_{r}}$ owing to the tracelessness (3.11) and transversality (3.13) of $F_{A_{1} \ldots A_{r}}$.

\subsection{Generating Functions}

Like for intrinsic tensors (exercise 1.2), it is useful employ an operator formalism and encode the ambient representatives of high-rank tensors in generating functions.

\section{Bulk fields}

For ambient representatives $\S 3.1$ of totally symmetric bulk fields, we have

$$
T_{A_{1} \ldots A_{s}}(X) \longrightarrow T(X, U)=\frac{1}{s !} T_{A_{1} \ldots A_{s}}(X) U^{A_{1}} \ldots U^{A_{s}}
$$


with constant $(d+2)$-dimensional ambient auxiliary vector $U^{A}$. For traceless fields, we may replace $U \rightarrow W$, with $W^{2}=0$.

Like for the intrinsic case, the covariant derivative (3.8) also gets modified in the generating function formalism. It takes the form

$$
\nabla_{A}=\mathscr{P}_{A}^{B} \frac{\partial}{\partial X^{B}}-\frac{X^{B}}{X^{2}} \Sigma_{A B}, \quad X \cdot \nabla=0
$$

where

$$
\Sigma_{A B}=U_{[A} \frac{\partial}{\partial U^{B]}}=U_{A} \frac{\partial}{\partial U^{B}}-U_{B} \frac{\partial}{\partial U^{A}},
$$

is the spin operator in the ambient generating function formalism.

In this case we have the operator algebra

$$
\left[X \cdot \partial_{U}, \nabla_{A}\right]=0, \quad\left[\partial_{U} \cdot \partial_{U}, \nabla_{A}\right]=0, \quad\left[\nabla_{A}, X^{2}\right]=0 .
$$

Exercise 4.1: Homogeneity degree

To demonstrate the power of the operator formalism, let's derive the homogeneity condition

(3.7) on harmonic (3.6) ambient representatives of totally symmetric fields.

Using that, in this totally symmetric case,

$$
i J_{A B}=X_{[A} \partial_{B]}^{X}+U_{[A} \partial_{B]}^{U},
$$

derive the relation

$$
\frac{1}{2} J_{A B} J^{A B}=\left(U \cdot \partial_{U}\right)\left(U \cdot \partial_{U}+d-2\right)+\left(X \cdot \partial_{X}\right)\left(d+X \cdot \partial_{X}\right)-X^{2} \partial_{X}^{2} .
$$

For a field carrying the module $\mathfrak{s} o(d, 2)$ module $\mathscr{D}(\Delta, s)$ represented by the ambient tensor $T_{A_{1} \ldots A_{s}}$, we have (see $\$ 1.3$ )

$$
\frac{1}{2} J_{A B} J^{A B} T(X, U)=(\Delta(\Delta-d)+s(s+d-2)) T(X, U) .
$$

Using (3.23), show that

$$
\partial_{X}^{2} T(X, U) \quad \Longrightarrow \quad\left(X \cdot \partial_{X}-\mu\right) T(X, U)=0, \quad \text { with } \quad \mu=\Delta \quad \text { or } \quad d-\Delta .
$$

\section{Boundary fields}

For representatives $\S 1.2$ of traceless and totally symmetric boundary fields, we have

$$
F_{A_{1} \ldots A_{r}}(P) \longrightarrow F(P, Z)=\frac{1}{r !} F_{A_{1} \ldots A_{r}}(P) Z^{A_{1}} \ldots Z^{A_{r}}, \quad Z^{2}=0 .
$$

The tangentiality condition (3.13), expressed in the operator formalism as,

$$
\left(P \cdot \frac{\partial}{\partial Z}\right) F(P, Z)=0
$$


can be enforced by demanding $F(P, Z+\alpha P)=F(P, Z)$, for any $\alpha$. The extra redundancy (3.14) is carried by the orthogonality condition $Z \cdot P=0$.

The ambient auxiliary vector $Z$ is related to the intrinsic one $z$ (introduced in exercise 3.1 ) via

$$
Z^{A}=z^{i} \frac{\partial P^{A}}{\partial y^{i}}=z^{i}\left(y_{i}, \delta_{i}^{j},-y_{i}\right)=\left(z \cdot y, z^{j},-z \cdot y\right) .
$$

\section{Higher Spin Interactions from CFT}

Let's make more precise how we extract interactions in higher-spin gauge theories from CFT. Assuming the existence of a fully non-linear action principle $S_{\mathrm{HS}}$ for a theory of higher-spin gauge fields, we perform a weak-field expansion around an empty AdS background in powers of the field fluctuations (which we denote collectively by $\varphi_{i}$ )

$$
S_{\mathrm{HS} \text { AdS }}=G S_{\mathrm{HS} \text { AdS }}^{(2)}\left[\varphi_{i}\right]+G^{3 / 2} S_{\mathrm{HS} \text { AdS }}^{(3)}\left[\varphi_{i}\right]+G^{2} S_{\mathrm{HS} \text { AdS }}^{(4)}\left[\varphi_{i}\right]+\ldots,
$$

where $S_{\mathrm{HS} \text { AdS }}^{(n)}$ is order- $n$ in the field fluctuations about empty AdS. As we saw in $\S 1.4$, the kinetic term of spin- $s$ gauge field $\varphi_{s}$ is given by the Fronsdal action

$$
S_{\mathrm{HS} \mathrm{AdS}}^{(2)}\left[\varphi_{s}\right]=\frac{s !}{2} \int_{\operatorname{AdS}_{d+1}} \varphi_{s}\left(x ; \partial_{u}\right)\left(1-\frac{1}{4} u^{2} \partial_{u} \cdot \partial_{u}\right) \mathscr{F}_{s}\left(x ; u, \nabla, \partial_{u}\right) \varphi_{s}(x, u),
$$

where $\mathscr{F}_{s}$ is the Fronsdal operator

$$
\begin{aligned}
\mathscr{F}_{s}\left(x, u, \nabla, \partial_{u}\right) & =\square-m_{s}^{2}-u^{2}\left(\partial_{u} \cdot \partial_{u}\right)-(u \cdot \nabla)\left(\left(\nabla \cdot \partial_{u}\right)-\frac{1}{2}(u \cdot \nabla)\left(\partial_{u} \cdot \partial_{u}\right)\right), \\
m_{s}^{2} R^{2} & =(s+d-2)(s-2)-s .
\end{aligned}
$$

The question is then the existence of non-trivial interaction terms $S_{\mathrm{HS} \text { AdS }}^{(n)}$ with $n>2$. In the context of holography, from the GKP/W formula the possibility of consistent interacting theories of higher-spin gauge fields on an AdS background appears to be quite natural:

We have (2.5)

$$
\exp \left(-F_{\text {free CFT }}[\bar{\varphi}]\right)=\int_{\left.\varphi\right|_{\partial \mathrm{AdS}}=\bar{\varphi}} \mathscr{D} \varphi \exp \left(-\frac{1}{G} S_{\mathrm{HS} \text { AdS }}[\varphi]\right),
$$

where $F_{\text {free }}$ CFT is the generating function of connected correlators in the dual free CFT. ${ }^{27}$ From the relation (2.11) between the bulk coupling $g$ and $N_{\text {d.o.f }}$, at large $N_{\text {d.o.f }}$ we have

$$
\exp \left(-F_{\text {free CFT }}[\bar{\varphi}]\right)=\left.\exp \left(-\frac{1}{G} S_{\mathrm{HS} \text { AdS }}[\varphi]\right)\right|_{\left.\varphi\right|_{\partial \mathrm{AdS}}=\bar{\varphi}}=\left.\prod_{n=2}^{\infty} \exp \left(-\sqrt{G}^{n-2} S_{\mathrm{HS} \text { AdS }}^{(n)}[\varphi]\right)\right|_{\left.\varphi\right|_{\partial \mathrm{AdS}}=\bar{\varphi}}
$$

\footnotetext{
${ }^{27}$ In fact, for free CFTs the $1 / N_{\text {d.o.f }}$ expansion is exact,

$$
F_{\text {free CFT }}=N_{\text {dof }} F_{\text {free CFT }}^{(0)} .
$$
}


That $F_{\text {free CFT }}$ is non-trivial indicates, via (4.6), non-trivial interactions in the higher-spin gauge theory on $\mathrm{AdS}_{d+1}$. With the knowledge of $F_{\text {free CFT, }}$, which is straightforward to determine in a free CFT, we may use (4.6) to iteratively extract metric-like interactions in the dual higher-spin gauge theory:

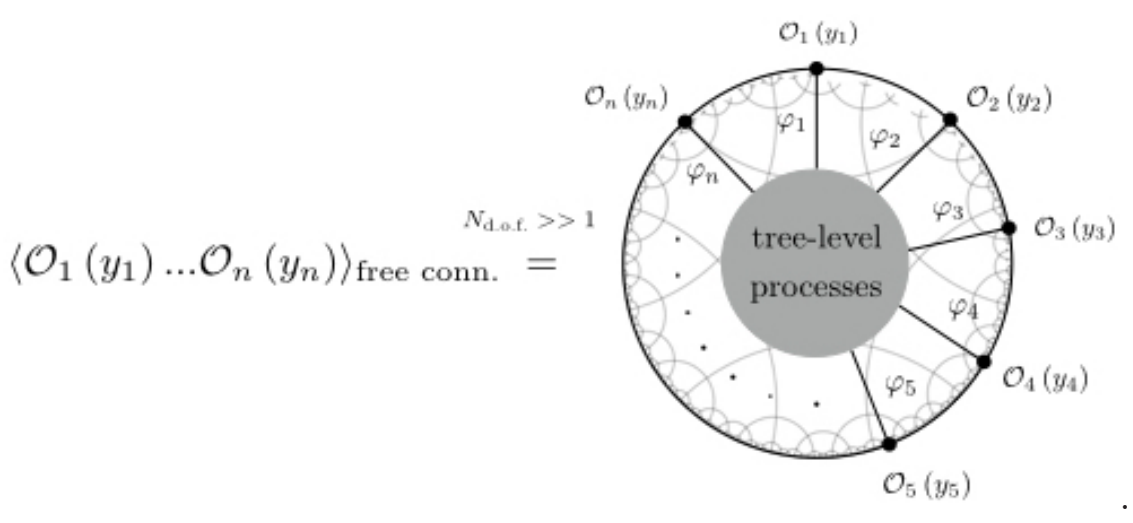

To this end a systematic approach needs to be developed to compute tree-level Feynman diagrams in AdS, known as Witten diagrams, for theories containing an infinite number of higher-spin gauge fields. This is the focus of the following sections.

\subsection{Witten Diagrams in Higher Spin Theories}

\subsubsection{Warm-up: Scalar Fields in AdS}

For simplicity, for the remainder of these notes we set the AdS radius $R=1$.

To lay down the basics of evaluating tree-level Witten diagrams using the ambient space formalism, let's begin with the simplest example of a scalar field theory in AdS. In this way we are free from the extra complexity added when considering fields of higher-spin.

Consider the action

$$
S\left[\varphi_{i}\right]=\frac{1}{G} \int_{\text {AdS }} \frac{1}{2} \nabla_{\mu} \varphi_{i} \nabla^{\mu} \varphi_{i}+m_{i}^{2} \varphi_{i}^{2}+g \varphi_{1} \varphi_{2} \varphi_{3}+\ldots, \quad i=1,2,3,
$$

with

$$
m_{i}^{2}=\Delta_{i}\left(\Delta_{i}-d\right) .
$$

In accordance with the GKP/W formula, at weak coupling the generating function of connected correlators at large $N_{\text {d.o.f }}$ in the dual CFT is given holographically by the on-shell action, subject to the boundary conditions

$$
\lim _{z \rightarrow 0} \varphi_{i}(z, y) z^{\Delta_{i}-d}=\bar{\varphi}_{i}(y) .
$$

The first step is to solve the equations of motion,

$$
\frac{\delta S}{\delta \varphi_{i}}=\left(-\square+m_{i}^{2}\right) \varphi_{i}+g \varphi_{j} \varphi_{k}+\ldots=0, \quad i \neq j \neq k
$$

subject to (4.10). Since we are at weak coupling, this can be solved perturbatively in the boundary values $\bar{\varphi}$ using integral kernels. We expand

$$
\varphi_{i}(x)=\varphi_{i}^{(0)}(x)+\varphi_{i}^{(1)}(x)+\varphi_{i}^{(2)}(x)+\ldots,
$$


where $\varphi_{i}^{(n)}$ is the solution at order $n+1$ in the $\bar{\varphi}$. This gives rise to the system of equations

$$
\begin{aligned}
\left(-\square+m_{i}^{2}\right) \varphi_{i}^{(0)} & =0, \\
\left(-\square+m_{i}^{2}\right) \varphi_{i}^{(1)}+g \varphi_{j}^{(0)} \varphi_{k}^{(0)} & =0, \\
\left(-\square+m_{i}^{2}\right) \varphi_{i}^{(2)}+g \varphi_{j}^{(0)} \varphi_{k}^{(1)}+g \varphi_{j}^{(1)} \varphi_{k}^{(0)} & =0,
\end{aligned}
$$

to be solved order-by-order in the $\bar{\varphi}$.

The solution of the first, linear, equation

$$
\left(-\square+m^{2}\right) \varphi_{i}^{(0)}=0,
$$

can be constructed from the boundary data using the corresponding bulk-to-boundary propagator. This the integral kernel

$$
\varphi_{i}^{(0)}(z, y)=\int_{\partial \mathrm{AdS}} d^{d} y^{\prime} K_{\Delta_{i}}(z, y ; \bar{y}) \bar{\varphi}_{i}(\bar{y})
$$

where $^{28}$

$$
\left(-\square+m_{i}^{2}\right) K_{\Delta_{i}}(z, y ; \bar{y})=0, \quad \lim _{z \rightarrow 0}\left(z^{\Delta_{i}-d} K_{\Delta_{i}}(z, y ; \bar{y})\right)=\frac{1}{2 \Delta_{i}-d} \delta^{d}(y-\bar{y}) .
$$

Higher-order solutions require the bulk-to-bulk propagator

$$
\left(-\square+m_{i}^{2}\right) \Pi_{\Delta_{i}}\left(x ; x^{\prime}\right)=\frac{1}{\sqrt{|g|}} \delta^{d+1}\left(x-x^{\prime}\right) .
$$

In this way we obtain the formal solution

$$
\begin{aligned}
\varphi_{i}^{(0)}(x) & =\int_{\partial \mathrm{AdS}} d^{d} y^{\prime} K_{\Delta_{i}}(z, y ; \bar{y}) \bar{\varphi}_{i}(\bar{y}), \\
\varphi_{i}^{(1)}(x) & =-g \int_{\mathrm{AdS}} d^{d+1} x^{\prime} \Pi_{\Delta_{i}}\left(x ; x^{\prime}\right) \varphi_{j}^{(0)}\left(x^{\prime}\right) \varphi_{k}^{(0)}\left(x^{\prime}\right), \\
\varphi_{i}^{(2)}(x) & =-g \int_{\mathrm{AdS}} d^{d+1} x^{\prime} \Pi_{\Delta_{i}}\left(x ; x^{\prime}\right) \varphi_{j}^{(0)}\left(x^{\prime}\right) \varphi_{k}^{(1)}\left(x^{\prime}\right)-g \int_{\mathrm{AdS}} d^{d+1} x^{\prime} \Pi_{\Delta_{i}}\left(x ; x^{\prime}\right) \varphi_{j}^{(1)}\left(x^{\prime}\right) \varphi_{k}^{(0)}\left(x^{\prime}\right) \\
& \vdots
\end{aligned}
$$

What remains is to insert the form of the kernels and perform the integration over $\operatorname{AdS}_{d+1}$. The on-shell action is thus given by the diagrammatic expansion

$$
S\left[\left.\varphi_{i}\right|_{\partial \mathrm{AdS}}=\bar{\varphi}_{i}\right]=\frac{1}{2} \longrightarrow+\longrightarrow+\cdots
$$

\footnotetext{
${ }^{28}$ The seemingly out of place factor of $2 \Delta_{i}-d$ ensures consistency with the boundary limit of the bulk-to-bulk propagator (4.17).
} 
Connected correlation functions of the CFT operators $\mathscr{O}_{i}$ dual to the $\varphi_{i}$ can be computed at large $N_{\text {d.o.f }}$ by functionally differentiating (4.19) with respect to boundary values (sources) $\bar{\varphi}_{i}$.

In these lectures we restrict to the holographic computation of three-point functions at large $N_{\text {d.o.f }}$,

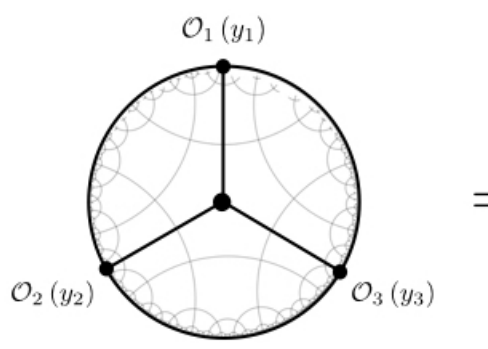

$$
=(-1)^{n} \frac{1}{G} \frac{\delta}{\delta \bar{\varphi}_{3}\left(y_{3}\right)} \frac{\delta}{\delta \bar{\varphi}_{2}\left(y_{2}\right)} \frac{\delta}{\delta \bar{\varphi}_{1}\left(y_{1}\right)} S\left[\left.\varphi_{i}\right|_{\partial \mathrm{AdS}}=\bar{\varphi}_{i}\right]
$$

which are generated by cubic interactions in AdS. To evaluate the corresponding Witten diagrams, in this case we just require the bulk-to-boundary propagators (4.16). In the Poincaré patch they are given by [27]

$$
K_{\Delta}(z, y ; \bar{y})=C_{\Delta, 0}\left(\frac{z}{z^{2}+\left(y-y^{\prime}\right)^{2}}\right)^{\Delta}, \quad C_{\Delta, 0}=\frac{\Gamma(\Delta)}{2 \pi^{d / 2} \Gamma\left(\Delta+1-\frac{d}{2}\right)},
$$

where the near-boundary behaviour (4.16) fixes the overall coefficient.

\section{Box 5.1: Fixing the Propagator normalisation}

Here we show explicitly how the propagator normalisation (4.21) is fixed by the near-boundary behaviour (4.16). At the linearised level, we have

$$
\varphi(z, y)=\int d^{d} \bar{y} K_{\Delta, 0}(z, y ; \bar{y}) \bar{\varphi}(\bar{y}) .
$$

Setting for simplicity $y=0$ by translation invariance and going to radial co-ordinates $\rho=|\bar{y}|$, we have

$$
\begin{aligned}
\int d^{d} \bar{y} K_{\Delta, 0}(z, 0 ; \bar{y}) \bar{\varphi}(\bar{y}) & =z^{\Delta} \Omega_{d-1} \int d \rho C_{\Delta, 0} \frac{\rho^{d-1}}{\left(z^{2}+\rho^{2}\right)^{\Delta}} \bar{\varphi}(\rho) \\
& =z^{d-\Delta} \Omega_{d-1} \int d t C_{\Delta, 0} \frac{t^{d-1}}{\left(1+t^{2}\right)^{\Delta}} \bar{\varphi}(t z)
\end{aligned}
$$

where $\Omega=\frac{2 \pi^{d / 2}}{\Gamma(d / 2)}$ and in the second equality we made the change of variables $t=\rho / z$. Then, expanding $\bar{\varphi}(t z)$ about $z=0$,

$$
\begin{aligned}
\lim _{z \rightarrow 0} \varphi(z, 0) z^{\Delta-d} & =C_{\Delta, 0} \Omega_{d-1} \int d t \frac{t^{d-1}}{\left(1+t^{2}\right)^{\Delta}} \bar{\varphi}(0)+\mathscr{O}(z) \\
& =C_{\Delta, 0} \frac{\pi^{d / 2}}{\Gamma(d / 2)} \frac{\Gamma(d / 2) \Gamma(\Delta-d / 2)}{\Gamma(\Delta)} .
\end{aligned}
$$


To obtain the boundary behaviour (4.10) we therefore require

$$
C_{\Delta, 0}=\frac{\Gamma(\Delta)}{2 \pi^{d / 2} \Gamma\left(\Delta+1-\frac{d}{2}\right)} .
$$

In the following we demonstrate how to evaluate Witten diagrams (4.20) using ambient space techniques $\S 3$, which admit a straightforward generalisation to the higher-spin case. In the ambient language, the propagator (4.21) takes the simple form

$$
K_{\Delta}(X ; P)=\frac{C_{\Delta, 0}}{(-2 X \cdot P)^{\Delta}}
$$

with bulk and boundary points

$$
X=\left(\frac{z^{2}+y^{2}+1}{2 z}, \frac{y^{i}}{z}, \frac{1-z^{2}-y^{2}}{2 z}\right), \quad P=\left(\frac{1}{2}\left(1+\bar{y}^{2}\right), \bar{y}^{i}, \frac{1}{2}\left(1-\bar{y}^{2}\right)\right) .
$$

At large $N_{\text {d.o.f. }}$, we have

$$
\left\langle\mathscr{O}_{1}\left(y_{1}\right) \mathscr{O}_{2}\left(y_{2}\right) \mathscr{O}_{3}\left(y_{3}\right)\right\rangle=g \int_{\text {AdS }} d X K_{\Delta_{1}}\left(X ; P_{1}\right) K_{\Delta_{2}}\left(X ; P_{2}\right) K_{\Delta_{3}}\left(X ; P_{3}\right)
$$

Evaluating the bulk integral can be dramatically simplified by using the Schwinger-parameterised form for the propagator $[97,99]^{29}$

$$
K_{\Delta}(X ; P)=\frac{C_{\Delta, 0}}{\Gamma(\Delta)} \int_{0}^{\infty} \frac{d t}{t} t^{\Delta} e^{2 t P \cdot X}
$$

In this way we have

$$
\begin{aligned}
g \int_{\mathrm{AdS}} d X K_{\Delta_{1}}\left(X ; P_{1}\right) K_{\Delta_{2}}\left(X ; P_{2}\right) K_{\Delta_{3}}\left(X ; P_{3}\right) \\
=g \int_{0}^{\infty} \prod_{i=1}^{3}\left(\frac{C_{\Delta_{i}, 0}}{\Gamma\left(\Delta_{i}\right)} \frac{d t_{i}}{t_{i}} t^{\Delta_{i}}\right) \int_{\mathrm{AdS}} d X e^{2\left(t_{1} P_{1}+t_{2} P_{2}+t_{3} P_{3}\right) \cdot X} \\
=g \pi^{\frac{d}{2}} \Gamma\left(\frac{-d+\sum_{i=1}^{3} \Delta_{i}}{2}\right) \int_{0}^{\infty} \prod_{i=1}^{3}\left(\frac{C_{\Delta_{i}, 0}}{\Gamma\left(\Delta_{i}\right)} \frac{d t_{i}}{t_{i}} t_{i}^{\Delta_{i}}\right) e^{\left(-t_{1} t_{2} P_{12}-t_{1} t_{3} P_{13}-t_{2} t_{3} P_{23}\right)},
\end{aligned}
$$

where we used box 5.2 to evaluate the bulk integral and defined $P_{i j}=-2 P_{i} \cdot P_{j}$. Through the change of variables,

$$
t_{1}=\sqrt{\frac{m_{2} m_{3}}{m_{1}}}, \quad t_{2}=\sqrt{\frac{m_{1} m_{3}}{m_{2}}}, \quad t_{3}=\sqrt{\frac{m_{1} m_{2}}{m_{3}}},
$$

we obtain the final result

\footnotetext{
${ }^{29}$ This is straightforward to obtain using the integral form of the Gamma function
}

$$
\Gamma(t) \alpha^{-t}=\int_{0}^{\infty} \frac{d u}{u} u^{t} e^{-\alpha u} .
$$




$$
\begin{aligned}
\left\langle\mathscr{O}_{1}\left(y_{1}\right) \mathscr{O}_{2}\left(y_{2}\right) \mathscr{O}_{3}\right. & \left.\left(y_{3}\right)\right\rangle \\
& =\frac{1}{2} g \pi^{\frac{d}{2}} \Gamma\left(\frac{-d+\sum_{i=1}^{3} \Delta_{i}}{2}\right) \int_{0}^{\infty} \prod_{i=1}^{3}\left(\frac{C_{\Delta_{i}, 0}}{\Gamma\left(\Delta_{i}\right)} \frac{d m_{i}}{m_{i}} m_{i}^{\frac{\Delta_{i}}{2}}\right) \exp \left(-m_{i} P_{j k}\right) \\
& =g \mathrm{C}\left(\Delta_{1}, \Delta_{2}, \Delta_{3} ; 0\right) \frac{1}{P_{13}^{\frac{\Delta_{1}+\Delta_{3}-\Delta_{2}}{2}} P_{23}^{\frac{\Delta_{2}+\Delta_{3}-\Delta_{1}}{2}} P_{12}^{\frac{\Delta_{1}+\Delta_{2}-\Delta_{3}}{2}}} .
\end{aligned}
$$

where we introduced

$$
\begin{aligned}
& \mathrm{C}\left(\Delta_{1}, \Delta_{2}, \Delta_{3} ; 0\right) \\
& \quad=\frac{1}{2} \pi^{\frac{d}{2}} \Gamma\left(\frac{-d+\sum_{i=1}^{3} \Delta_{i}}{2}\right) C_{\Delta_{1}, 0} C_{\Delta_{2}, 0} C_{\Delta_{3}, 0} \frac{\Gamma\left(\frac{\Delta_{1}+\Delta_{2}-\Delta_{3}}{2}\right) \Gamma\left(\frac{\Delta_{1}+\Delta_{3}-\Delta_{2}}{2}\right) \Gamma\left(\frac{\Delta_{2}+\Delta_{3}-\Delta_{1}}{2}\right)}{\Gamma\left(\Delta_{1}\right) \Gamma\left(\Delta_{2}\right) \Gamma\left(\Delta_{3}\right)} .
\end{aligned}
$$

Box 5.2: Tree-level contact diagrams using Schwinger Parameterisation

Consider the $n$-point contact diagram generated by the vertex involving scalars $\varphi_{i}$

$$
V_{12 \ldots n}=g \varphi_{1} \varphi_{2} \ldots \varphi_{n},
$$

Using the Schwinger-parameterised form of the bulk-to-boundary propagators (4.30) one encounters the bulk integral

$$
\mathscr{A}^{\text {cont. }}\left(y_{1}, \ldots, y_{n}\right)=\left(\prod_{i=1}^{n} C_{\Delta_{i}, 0}\right) \int_{0}^{+\infty} \prod_{i=1}^{n}\left(\frac{d t_{i}}{t_{i}} t^{\Delta_{i}}\right) \int_{\mathrm{AdS}} d X \exp \left(2 \sum_{i=1}^{n} t_{i} P_{i} \cdot X\right)
$$

which is the extension of (4.31) to $n>3$. Defining $T=\sum_{i=1}^{n} t_{i} P_{i}$, by Lorentz invariance we may simply choose $T=|T|(1, \mathbf{0}, 0)$. Like this we obtain

$$
\begin{aligned}
\int_{\operatorname{AdS}} d X \exp \left(2 \sum_{i=1}^{n} t_{i} P_{i} \cdot X\right) & =\int_{0}^{+\infty} \frac{d z}{z} z^{-d} \int d^{d} y e^{-\left(1+z^{2}+y^{2}\right)|T| / z} \\
& =\pi^{d / 2} \int_{0}^{+\infty} \frac{d z}{z} z^{-d / 2} e^{-\left(z-T^{2} / z\right)}
\end{aligned}
$$

where in the second line we evaluated the Gaussian integral over $y .{ }^{30}$ Returning to the full (4.36) and rescaling $t_{i} \rightarrow t_{i} / \sqrt{z}$, we can evalute the final integral over $z$ by using the integral representation of the Gamma function (see footnote 29.)

$$
\begin{aligned}
\pi^{d / 2} \int_{0}^{+\infty} \prod_{i=1}^{n}\left(\frac{d t_{i}}{t_{i}} t^{\Delta_{i}}\right) \int_{0}^{+\infty} \frac{d z}{z} z^{-d / 2} e^{-\left(z-T^{2} / z\right)} \\
=\pi^{d / 2} \int_{0}^{+\infty} \prod_{i=1}^{n}\left(\frac{d t_{i}}{t_{i}} t^{\Delta_{i}}\right) e^{T^{2}} \int_{0}^{+\infty} \frac{d z}{z} z^{-\frac{d}{2}+\frac{1}{2} \sum_{i=1}^{n} \Delta_{i}} e^{-z} \\
=\pi^{d / 2} \Gamma\left(-\frac{d}{2}+\frac{1}{2} \sum_{i=1}^{n} \Delta_{i}\right) \int_{0}^{+\infty} \prod_{i=1}^{n}\left(\frac{d t_{i}}{t_{i}} t^{\Delta_{i}}\right) e^{-\sum_{i<k} t_{i} t_{k} P_{i k}}
\end{aligned}
$$

where we used $T^{2}=\sum_{i<k} t_{i} t_{k} P_{i k}$ 


\subsubsection{Witten Diagrams with External HS Fields}

With the basics in place, we now turn to the case of external higher-spin fields. One of the main virtues of the ambient space approach is that it makes the $S O(d, 2)$ symmetry manifest. That the ambient representative fields are homogeneous in both the bulk and boundary co-ordinates allows us to straightforwardly express a given Witten diagram with spinning external legs as some differential operator $\mathscr{F}\left(P_{i}, \partial_{P_{i}}\right)$ acting on a diagram with only external scalars, as illustrated below for tree-level three-point Witten diagrams
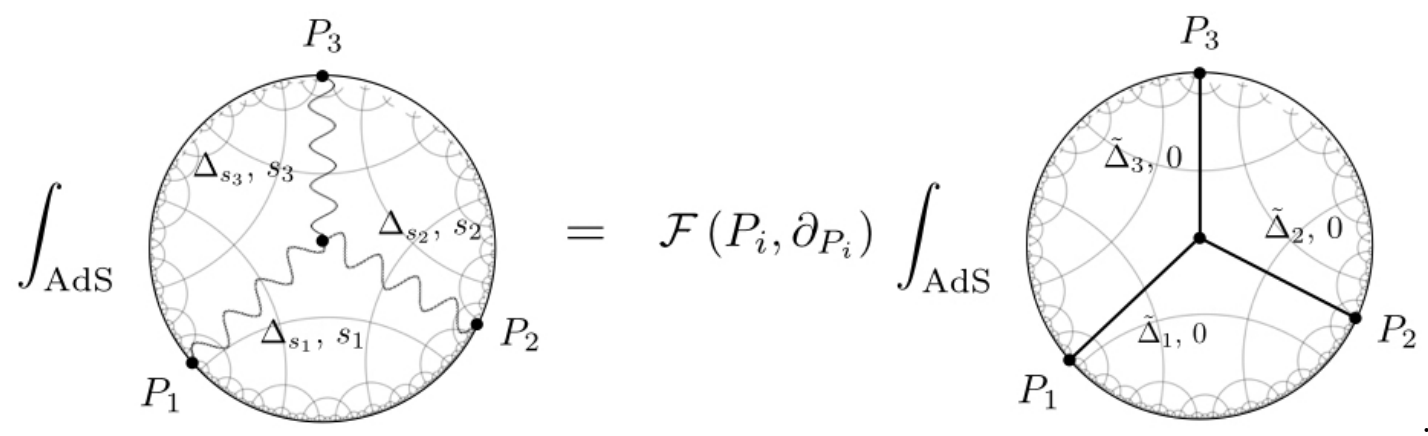

(4.41)

In particular, we can reduce a three-point Witten diagram involving external spinning fields to one of the type (4.28), as we explain in the following.

\section{Spinning Bulk-to-Boundary Propagators}

For a totally symmetric and traceless spin-s field of energy $\Delta$, the corresponding bulk-toboundary propagator satisfies the wave equation [157]

$$
\begin{aligned}
& (-\square+\Delta(\Delta-d)-s) K_{\Delta, \mu_{1} \ldots \mu_{s}} i_{1} \ldots i_{s}\left(z, y ; y^{\prime}\right)=0,
\end{aligned}
$$

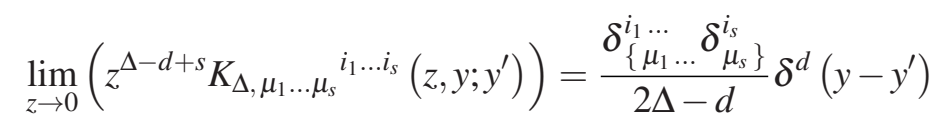

The $S O(d, 2)$ symmetry places constraints on the ambient representative

$$
K_{\Delta, s}\left(X, \alpha_{1} W ; \lambda P, \alpha_{2} Z+\beta P\right)=\lambda^{-\Delta}\left(\alpha_{1} \alpha_{2}\right)^{s} K_{\Delta, s}(X, W ; P, Z)
$$

which fix its structure uniquely: ${ }^{31}$

$$
K_{\Delta, s}(X, W ; P, Z)=(W \cdot \mathscr{P} \cdot Z)^{s} \frac{C_{\Delta, s}}{(-2 X \cdot P)^{\Delta}}
$$

$$
\begin{aligned}
& { }^{30} \text { In particular, } \\
& \qquad \int d^{d} y e^{-y^{2}|T| / z}=\left(\frac{z}{|T|}\right)^{d / 2} \pi^{d / 2} . \\
& { }^{31} \text { Since tracelessness in the bulk indices in this case is ensured by } \\
& \qquad P^{2}=0, \quad Z^{2}=0, \quad Z \cdot P=0,
\end{aligned}
$$

we may replace $W \rightarrow U$, with $U^{2} \neq 0$. 
The projector

$$
\mathscr{P}^{A}{ }_{B}=\delta_{B}^{A}-\frac{P^{A} X_{B}}{P \cdot X}
$$

ensures transversality of the propagator at both its bulk and boundary points, while the overall coefficient $C_{\Delta, s}$ is fixed by equation (4.43)

$$
C_{\Delta, s}=\frac{(s+\Delta-1) \Gamma(\Delta)}{2 \pi^{d / 2}(\Delta-1) \Gamma\left(\Delta+1-\frac{d}{2}\right)} .
$$

The latter also fixes the normalisation of the two-point function on the boundary

$$
\left\langle\mathscr{O}_{\Delta, s}\left(P_{1}\right) \mathscr{O}_{\Delta, s}\left(P_{2}\right)\right\rangle=K_{\Delta, s}\left(P_{1} ; P_{2}\right)
$$

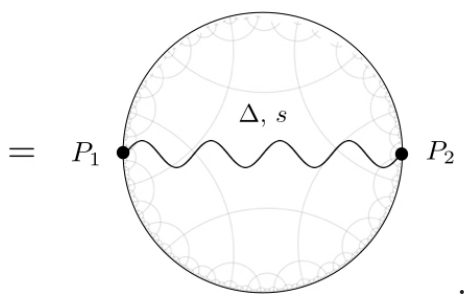

\section{Spinning Witten diagrams from Scalar Witten diagrams}

The trick is that we can express spinning propagators and their derivatives in the form

$$
\left(U_{2} \cdot \partial_{X}\right)^{n} K_{\Delta, s}\left(X, U_{1} ; P, Z\right)=\mathscr{G}\left(P, \partial_{P} ; Z, U_{1}, U_{2}\right) K_{\Delta+n, 0}(X ; P),
$$

for some function $\mathscr{G}$ acting on a scalar bulk-to-boundary propagator, which we now determine. It is sufficient to consider the $n=0$ and $s=0$ cases:

For $n=0$ it is straightforward to confirm that

$$
K_{\Delta, s}(X, U ; P, Z)=\frac{1}{(\Delta-1)_{s}}\left(\mathscr{D}_{P}(Z ; U)\right)^{s} K_{\Delta, 0}(X ; P),
$$

with

$$
\mathscr{D}_{P}(Z ; U)=(Z \cdot U)\left(Z \cdot \frac{\partial}{\partial Z}-P \cdot \frac{\partial}{\partial P}\right)+(P \cdot U)\left(Z \cdot \frac{\partial}{\partial Z}\right),
$$

implementing the projection (4.47).

For $s=0$ we have

$$
\left(U \cdot \partial_{X}\right)^{n} K_{\Delta, 0}(X ; P)=2^{n}(\Delta)_{n}(U \cdot P)^{n} K_{\Delta+n, 0}(X ; P),
$$

which together with (4.51) fixes (4.50).

With the form (4.50) of bulk-to-boundary propagators and their derivatives, any Witten diagram with spinning external fields may be expressed in terms of one with only external scalars, with no derivatives acting upon them. For tree-level three-point Witten diagrams, this in particular means that we can recycle the result (4.33) we previously derived for the three-point Witten diagram generated by the basic cubic vertex (4.8) for scalar fields. This is illustrated for a three-point Witten diagram involving a single external field of arbitrary spin in the following. 


\section{Example: Three-point Witten Diagrams with Single HS Field}

We now demonstrate the approach described in the previous section for the simplest example of the tree-level three-point Witten diagram generated by a cubic interaction between two scalars $\phi_{1} \& \phi_{2}$ of energies $\Delta_{1,2}$ and a totally symmetric spin-s field $\varphi_{s}$ of energy $\Delta_{s}$. This type of vertex is unique on-shell ${ }^{32}$ up to an overall coupling [158], and takes the form ${ }^{33}$

$$
\hat{\mathscr{V}}_{0,0, s}=g \varphi_{\mu_{1} \ldots \mu_{s}} \phi_{1} \nabla^{\mu_{1}} \ldots \nabla^{\mu_{s}} \phi_{2}=s ! g \phi_{1}(x)\left(\partial_{u} \cdot \nabla\right)^{s} \phi_{2}(x) \varphi_{s}(x, u),
$$

for some coupling $g$. In the second equality we expressed the vertex in the operator formalism (exercise 1.2).
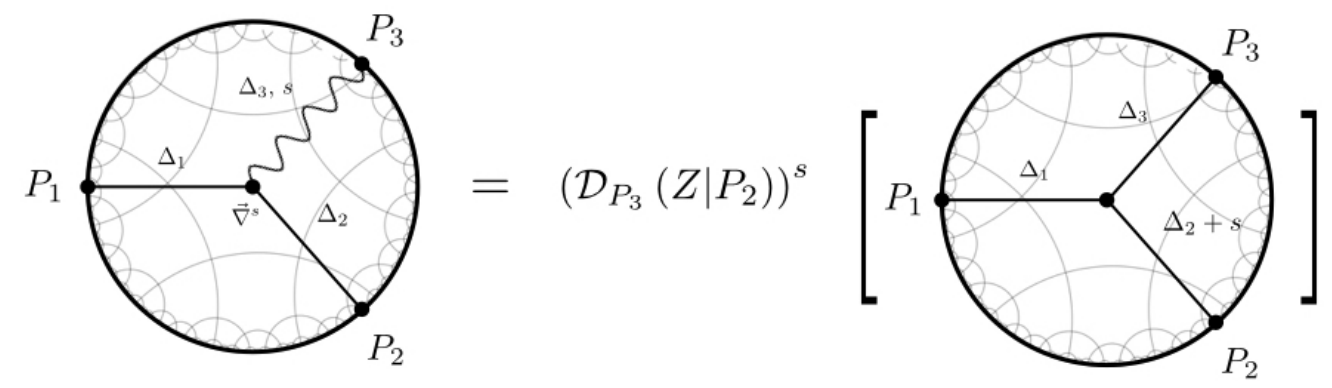

Figure 3:

To apply the methods of the previous section to evaluate the corresponding tree-level Witten diagram, we have first to re-write the vertex (4.54) in ambient space. Following the dictionary in $\S 3$, this is simply

$$
\hat{\mathscr{V}}_{0,0, s}(X)=g \varphi_{A_{1} \ldots A_{s}} \phi_{1} \nabla^{A_{1}} \ldots \nabla^{A_{s}} \phi_{2}=s ! g \phi_{1}(X)\left(\partial_{U} \cdot \nabla\right)^{s} \phi_{2}(X) \varphi_{s}(X, U),
$$

where we recall the homogeneity and tangentiality conditions $\$ 3.1$ on ambient representatives.

In fact in ambient space the vertex (4.55) can be simplified further: Terms which deform the ambient representative (3.19) of the covariant derivative from the $(d+2)$-dimensional flat partial derivative $\partial_{X}^{A}$ drop out, owing to the tracelessness and tangentiality of $\varphi_{S}(X, U)$,

$$
\partial_{U} \cdot \partial_{U} \varphi_{s}(X, U)=0, \quad X \cdot \partial_{U} \varphi_{s}(X, U)=0 .
$$

We therefore arrive to the very simple expression

$$
\hat{\mathscr{V}}_{s, 0,0}(X)=s ! g \phi_{1}(X)\left(\partial_{U} \cdot \partial_{X}\right)^{s} \phi_{2}(X) \varphi_{s}(X, U),
$$

whose tree-level three-point Witten diagram we now evaluate following the approach outlined in the previous section. According to the standard Feyman rules $\S 4.1 .1$, this is

\footnotetext{
${ }^{32}$ I.e. unique when enforcing the constraints (1.32) of the Fierz system, including the tracelessness and divergenceless constraints.

${ }^{33}$ This can also be understood from the perspective of a dual CFT, since there is only a single conformally covariant tensor structure that can be written down for a correlation function containing two scalar operators and a spin- $s$ operator.
} 


$$
\begin{aligned}
\left\langle\mathscr{O}_{\Delta_{1}, 0}\left(P_{1}\right) \mathscr{O}_{\Delta_{2}, 0}\left(P_{2}\right) \mathscr{O}_{\Delta_{3}, s}\right. & \left.\left(P_{3} ; Z\right)\right\rangle \\
& =s ! g \int_{\mathrm{AdS}} K_{\Delta_{3}, s}\left(X, \partial_{U} ; P_{3}, Z\right) K_{\Delta_{1}, 0}\left(X ; P_{1}\right)\left(U \cdot \partial_{X}\right)^{s} K_{\Delta_{2}, 0}\left(X ; P_{2}\right),
\end{aligned}
$$

where the dual CFT operators $\mathscr{O}_{\Delta_{1}}, \mathscr{O}_{\Delta_{2}, 0}$ and $\mathscr{O}_{\Delta_{s}, s}$ are sourced by $\phi_{1}, \phi_{2}$ and $\varphi_{s}$, respectively.

The core idea of $\S 4.1 .2$ was to generate such a Witten diagram with spinning external legs from the basic diagram (4.33) with only external scalars through the action of an appropriate differential operator in the boundary variables (equation (4.41)). With the simple form of the vertex (4.57), this is straightforward to attain using the expressions (4.51) and (4.53) for the bulk-to-boundary propagators. We get,

$$
\begin{aligned}
\left\langle\mathscr{O}_{\Delta_{1}, 0}\left(P_{1}\right) \mathscr{O}_{\Delta_{2}, 0}\left(P_{2}\right) \mathscr{O}_{\Delta_{3}, s}\left(P_{3} ; Z\right)\right\rangle & \\
& =\frac{s ! g}{\left(\Delta_{3}-1\right)_{s}}\left(\mathscr{D}_{P_{3}}\left(Z ; \partial_{U}\right)\right)^{s} \int_{\mathrm{AdS}} K_{\Delta_{3}, 0}\left(X ; P_{3}, Z\right) K_{\Delta_{1}, 0}\left(X ; P_{1}\right)\left(U \cdot \partial_{X}\right)^{s} K_{\Delta_{2}, 0}\left(X ; P_{2}\right) \\
& =\frac{s !\left(\Delta_{2}+1-\frac{d}{2}\right)_{s}}{\left(\Delta_{3}-1\right)_{s}}\left(\mathscr{D}_{P_{3}}\left(Z ; P_{2}\right)\right)^{s} g \int_{\mathrm{AdS}} K_{\Delta_{3}, 0}\left(X ; P_{3}, Z\right) K_{\Delta_{1}, 0}\left(X ; P_{1}\right) K_{\Delta_{2}+s, 0}\left(X ; P_{2}\right) .
\end{aligned}
$$

This relationship is depicted in figure 3 .

All that remains is to plug in the result (4.33) for the basic scalar three-point Witten diagram and evaluate the action of the differential operator $\mathscr{D}_{P}$, to arrive to the final expression ${ }^{34}$

$$
\begin{aligned}
\left\langle\mathscr{O}_{\Delta_{1}, 0}\left(P_{1}\right) \mathscr{O}_{\Delta_{2}, 0}\left(P_{2}\right) \mathscr{O}_{\Delta_{3}, s}\left(P_{3} ; Z\right)\right\rangle & \\
= & g C\left(\Delta_{1}, \Delta_{2}, \Delta_{3} ; s\right) \frac{\left(\left(Z \cdot P_{1}\right) P_{23}-\left(Z \cdot P_{2}\right) P_{13}\right)^{s}}{P_{13}^{\frac{\Delta_{1}+\Delta_{3}-\Delta_{2}+s}{2}} P_{23}^{\frac{\Delta_{2}+\Delta_{3}-\Delta_{1}+s}{2}} P_{12}^{\frac{\Delta_{1}+\Delta_{2}-\Delta_{3}+s}{2}}},
\end{aligned}
$$

with

$$
\begin{aligned}
& C\left(\Delta_{1}, \Delta_{2}, \Delta_{3} ; s\right) \\
& \quad=\frac{2^{s}\left(1-\frac{d}{2}+\Delta_{2}\right)_{s}}{\left(\Delta_{3}-1\right)_{s}}\left(\frac{\Delta_{1}+\Delta_{3}-\Delta_{2}-s}{2}\right)_{s} \mathrm{C}\left(\Delta_{1}, \Delta_{2}+s, \Delta_{3} ; 0\right) \\
& \quad=C_{\Delta_{1}, 0} C_{\Delta_{2}, 0} C_{\Delta_{3}, s} \frac{2^{s} \pi^{\frac{d}{2}} \Gamma\left(\frac{\Delta_{1}+\Delta_{2}+\Delta_{3}-d+s}{2}\right) \Gamma\left(\frac{\Delta_{1}+\Delta_{2}-\Delta_{3}+s}{2}\right) \Gamma\left(\frac{\Delta_{1}+\Delta_{3}-\Delta_{2}+s}{2}\right) \Gamma\left(\frac{\Delta_{2}+\Delta_{3}-\Delta_{1}+s}{2}\right)}{2 \Gamma\left(\Delta_{1}\right) \Gamma\left(\Delta_{2}\right) \Gamma\left(\Delta_{3}+s\right)} .
\end{aligned}
$$

The result (4.61) has precisely the space-time dependence that is required by conformal symmetry (see §4.3.1). It can be expressed in more familiar intrinsic terms using that

$$
P_{i j}=\left(y_{i}-y_{j}\right)^{2}, \quad Z^{A}=\left(z \cdot y_{3}, z^{j},-z \cdot y_{3}\right), \quad Z \cdot P_{1}=z \cdot y_{13}, \quad Z \cdot P_{2}=z \cdot y_{23},
$$

\footnotetext{
${ }^{34}$ The action of $\mathscr{D}_{P}$ is given by

$$
\begin{aligned}
\left(\mathscr{D}_{P_{3}}\left(Z \mid P_{2}\right)\right)^{s} \frac{1}{P_{13}^{\frac{\Delta_{1}+\Delta_{3}-\Delta_{2}-s}{2}} P_{23}^{\frac{\Delta_{2}+\Delta_{3}-\Delta_{1}+s}{2}}} P_{12}^{\frac{\Delta_{1}+\Delta_{2}-\Delta_{3}+s}{2}} & \\
& =\left(\frac{\Delta_{1}+\Delta_{3}-\Delta_{2}-s}{2}\right) \frac{\left(\left(Z \cdot P_{1}\right) P_{23}-\left(Z \cdot P_{2}\right) P_{13}\right)^{s}}{s} P_{13}^{\frac{\Delta_{1}+\Delta_{3}-\Delta_{2}+s}{2}} P_{23}^{\frac{\Delta_{2}+\Delta_{3}-\Delta_{1}+s}{2}} P_{12}^{\frac{\Delta_{1}+\Delta_{2}-\Delta_{3}+s}{2}}
\end{aligned}
$$
}


to obtain

$$
\begin{aligned}
\left\langle\mathscr{O}_{\Delta_{1}, 0}\left(y_{1}\right) \mathscr{O}_{\Delta_{2}, 0}\left(y_{2}\right)\right. & \left.\mathscr{O}_{\Delta_{3}, s}\left(y_{3} ; z\right)\right\rangle \\
& =g \mathrm{C}\left(\Delta_{1}, \Delta_{2}, \Delta_{3} ; s\right) \frac{\left(\left(z \cdot y_{13}\right) y_{23}^{2}-\left(z \cdot y_{23}\right) y_{13}^{2}\right)^{s}}{\left(y_{13}^{2}\right)^{\frac{\Delta_{1}+\Delta_{3}-\Delta_{2}+s}{2}}\left(y_{23}^{2}\right)^{\frac{\Delta_{2}+\Delta_{3}-\Delta_{1}+s}{2}}\left(y_{12}^{2}\right)^{\frac{\Delta_{1}+\Delta_{2}-\Delta_{3}+s}{2}}} .
\end{aligned}
$$

This approach is straightforward to apply to the general case of external fields with arbitrary integer spin [34]. On the other hand the result (4.64) is already enough to fix all higher-spin cubic interactions involving a single field of arbitrary integer spin, which we now consider.

\subsection{Holographic Reconstruction of HS Cubic Vertices}

Now that we know how to compute three-point Witten diagrams with an external field of arbitrary integer spin, we can already holographically re-construct part of the cubic order action of the minimal bosonic higher-spin theory on $\mathrm{AdS}_{d+1}$, which was introduced in $\$ 2.2$.

For this set-up, the GKP/W formula $§ 2.1$ reads: ${ }^{3536}$

$$
\begin{aligned}
F_{\text {free } O(N)}\left[\bar{\varphi}_{s}\right] & \stackrel{N \gg 1}{=} \frac{1}{G} S_{\text {HS AdS }}\left[\left.\varphi_{s}\right|_{\partial \operatorname{AdS}}=\bar{\varphi}_{s}\right] \\
& =\int_{\operatorname{AdS}_{d+1}} \sum_{s \in 2 \mathbb{Z}} \frac{s !}{2} \varphi_{s}\left(x, \partial_{u}\right)\left(\square-m_{s}^{2}\right) \varphi_{s}(x, u)+\sum_{s_{3} \leq s_{2} \leq s_{1}} \mathscr{V}_{s_{1}, s_{2}, s_{3}}\left(\varphi_{s_{i}}\right)+\ldots
\end{aligned}
$$

where $F_{\text {free }} O(N)$ is the generating function of connected correlators in the $d$-dimensional free scalar $O(N)$ model and $S_{\mathrm{HS} \text { AdS }}$ is a would be non-linear action for the dual minimal bosonic higher-spin theory, expanded around $\mathrm{AdS}_{d+1}$. Note that the action is on-shell, hence the dropping of gaugedependent terms in the kinetic term from the off-shell Fronsdal action (4.2).

In particular, the formula (4.65) implies that cubic interactions between gauge fields of a given triplet of spins $s_{1}-s_{2}-s_{3}$ are fixed by the three-point function of conserved currents (2.28) of the same triplet of spins,

$$
\begin{aligned}
& \left\langle\mathscr{J}_{s_{1}}\left(y_{1}\right) \mathscr{J}_{s_{2}}\left(y_{2}\right) \mathscr{J}_{s_{3}}\left(y_{3}\right)\right\rangle \stackrel{N \gg 1}{=} \frac{\delta}{\delta \bar{\varphi}_{s_{3}}\left(y_{3}\right)} \frac{\delta}{\delta \bar{\varphi}_{s_{2}}\left(y_{2}\right)} \frac{\delta}{\delta \bar{\varphi}_{s_{1}}\left(y_{1}\right)} \int_{\operatorname{AdS}_{d+1}} \mathscr{V}_{s_{1}, s_{2}, s_{3}}\left(\varphi_{s_{i}}\right) \\
& =\int_{\mathrm{AdS}}^{\mathcal{J}_{s_{3}}\left(y_{3}\right)}
\end{aligned}
$$

\footnotetext{
${ }^{35}$ In the present case of vector models, $N_{\text {d.o.f. }}=N$.

${ }^{36}$ Recall that for spin-s gauge fields we have $m_{s}^{2} R^{2}=(s-2)(s+d-2)-s$.
} 
In practice one can proceed by making the most general ansatz for the vertex $\mathscr{V}_{s_{1}, s_{2}, s_{3}}$ and solving (4.66) for its form [34]. This approach is particularly successful because the CFT is free, and so the correlation functions are straightforwardly computed by Wick contracting.

For the $\mathscr{V}_{0,0, s}$ vertices, as discussed in $\S 4.1 .2$, the ansatz one can write down is unique up to an overall coefficient. We can therefore use the result of the previous section to determine the cubic interactions involving a single gauge field of arbitrary spin, which we undertake in the following.

\subsection{Example: Cubic order action for $0-0-s$ interactions}

The simple illustrative example of this approach, which employs the results derived in $§ 4.1 .2$, is to extract the on-shell cubic interactions between two scalars $\varphi_{0}$ and a spin-s gauge field $\varphi_{s}$ in the bulk action.

For consistency with the spin-s gauge transformations, the $\mathscr{V}_{0,0, s}$ vertices have the form

$$
\mathscr{V}_{0,0, s}=g_{0,0, s} ! J_{s}\left(x, \partial_{u}\right) \varphi_{s}(x, u), \quad\left(\partial_{u} \cdot \nabla\right) J_{s}(x, u) \approx 0,
$$

where $J_{s}$ is a spin-s conserved current bi-linear in $\varphi_{0}$. Its explicit form is most straightforward to work out in ambient space, as we demonstrate in exercise 5.1 below.

\section{Exercise 5.1: AdS Conserved Currents from Flat Space}

Recall that we already encountered some spin- $s$ conserved currents that are scalar bi-linears in $\S 2.2$, but in flat space. In fact using the ambient space formalism, we can use this result to construct the analogous currents in AdS:

Suppose that we have a symmetric rank-s tensor $I_{S}$ in ambient space, that is conserved with respect to the ambient partial derivative

$$
\left(\partial_{U} \cdot \partial_{X}\right) I_{S}(X, U) \approx 0
$$

Can $I_{s}$ also define a conserved current in AdS? Using ambient representative (3.19) for the covariant derivative on AdS, show that

$$
\begin{aligned}
\left(\partial_{U} \cdot \nabla\right) I_{S}(X, U)=\left(\partial_{U} \cdot \partial_{X}\right) & I_{S}(X, U)+\frac{U \cdot X}{X^{2}} \partial_{U}^{2} I_{S}(X, U) \\
& -\frac{X \cdot \partial_{U}}{X^{2}}\left[X \cdot \partial_{X}+U \cdot \partial_{U}+d\right] I_{S}(X, U)
\end{aligned}
$$

The first term vanishes due to conservation (4.68), while the final term sits in the kernel of the pull-back (3.1) onto the AdS manifold and can thus be neglected. Therefore $I_{S}$ also represents a conserved current in AdS if

$$
\left[X \cdot \partial_{X}+U \cdot \partial_{U}+d\right] I_{S}(X, U)=0
$$

This condition is precisely satisfied by the flat space current (2.26) that we encountered in exercise 3.1, but with $\phi^{a} \rightarrow \varphi_{0}$ :

$$
I_{s}(X, U)=i^{s} \sum_{k=0}^{s}(-1)^{k}\left(\begin{array}{l}
s \\
k
\end{array}\right)\left(U \cdot \partial_{X}\right)^{k} \varphi_{0}(X)\left(U \cdot \partial_{X}\right)^{s-k} \varphi_{0}(X),
$$


Where the ambient representative of the bulk scalar $\varphi_{0}$ satisfies ((3.6) and (3.7))

$$
\begin{aligned}
\partial_{X}^{2} \varphi_{0}(X) & =0 \\
\left(X \cdot \partial_{X}-2+d\right) \varphi_{0}(x) & =0 .
\end{aligned}
$$

Taking the expression (4.71) for the conserved current, the vertex (4.67) in ambient space reads

$$
\mathscr{V}_{0,0, s}=g_{0,0, s} s ! i^{s} \varphi_{s}\left(X, \partial_{U}\right) \sum_{k=0}^{s}(-1)^{k}\left(\begin{array}{l}
s \\
k
\end{array}\right)(U \cdot \partial)^{k} \varphi_{0}(X)(U \cdot \partial)^{s-k} \varphi_{0}(X) .
$$

On the other hand, recall in $\S 4.1 .2$ we argued that the structure of vertices involving two scalars and a spin-s field is unique on-shell. In other words, it must be that

$$
\mathscr{V}_{0,0, s} \approx \alpha \hat{\mathscr{V}}_{0,0, s}
$$

for some constant $\alpha$ and $\hat{\mathscr{V}}_{0,0, s}$ is the basic vertex (4.54). Indeed, integrating by parts and using the on-shell (Fierz-Pauli) conditions (1.32) one finds

$$
\mathscr{V}_{0,0, s} \approx 2^{s} \hat{\mathscr{V}}_{0,0, s}=g_{0,0, s} s ! 2^{s} i^{s} \varphi_{s}\left(X, \partial_{U}\right)(U \cdot \partial)^{s} \varphi_{0}(X) .
$$

Re-cycling the result (4.64) for the Witten diagram generated by the basic vertex $\hat{\mathscr{V}}_{0,0, s}$, from the bulk side we have

$$
\begin{aligned}
\left\langle\mathscr{O}\left(y_{1}\right) \mathscr{O}\left(y_{2}\right) \mathscr{J}_{s}\left(y_{3} ; z\right)\right\rangle & \stackrel{N \gg 1}{=} 2^{s} g_{0,0, s} \mathrm{C}(d-2, d-2, s+d-2 ; s) \frac{\left(\left(z \cdot y_{13}\right) y_{23}^{2}-\left(z \cdot y_{23}\right) y_{13}^{2}\right)^{s}}{\left(y_{13}^{2}\right)^{\frac{d}{2}-1+s}\left(y_{23}^{2}\right)^{\frac{d}{2}-1+s}\left(y_{12}^{2}\right)^{\frac{d}{2}-1}} .
\end{aligned}
$$

What remains is to compare (4.77) with the result as computed in CFT, to which we now turn.

\subsubsection{Correlators in CFT}

In a $\mathrm{CFT},{ }^{37}$ the conformal symmetry fixes the structure of the three-point function up to a collection of coefficients [161]. For the present case of three-point functions involving a single operator of non-zero spin, there is a unique structure compatible with conformal symmetry

$$
\left\langle\mathscr{O}\left(y_{1}\right) \mathscr{O}\left(y_{2}\right) \mathscr{J}_{s}\left(y_{3} ; z\right)\right\rangle=\mathrm{C}_{\mathscr{O} \mathscr{O}} \mathscr{J}_{s} \frac{\left(\left(z \cdot y_{13}\right) y_{23}^{2}-\left(z \cdot y_{23}\right) y_{13}^{2}\right)^{s}}{\left(y_{13}^{2}\right)^{\frac{d}{2}-1+s}\left(y_{23}^{2}\right)^{\frac{d}{2}-1+s}\left(y_{12}^{2}\right)^{\frac{d}{2}-1}} .
$$

For free theories, $\mathrm{C}_{\mathscr{O} \mathscr{O} \mathscr{J}_{s}}$ is straightforward to compute by Wick contracting. To this end, it is convenient to employ the generating function representation (2.28) for the spin- $s$ currents, yielding for the free scalar $O(N)$ model (see exercise 5.2)

$$
C_{\mathscr{J}_{s} \mathscr{O} O}=8 N\left[\frac{1+(-1)^{s}}{2}\right] \frac{2^{s}\left(\frac{d}{2}-1\right)_{s}(d-3)_{s}}{\Gamma(s+1)} .
$$

\footnotetext{
${ }^{37}$ We only discuss the CFT side briefly here. For reviews / lecture notes on Conformal Field Theory see [117, 159, $160]$.
} 
Exercise 5.2 Three-point function coefficient

Using the generating function representation (2.28) for the spin-s currents, show that

$$
\begin{aligned}
\left\langle\mathscr{O}\left(y_{1}\right) \mathscr{O}\left(y_{2}\right) \mathscr{J}_{s}\left(y_{3} ; z\right)\right\rangle & \\
= & \frac{8 N}{\Gamma\left(\frac{d}{2}-1\right)^{3}}\left[\frac{1+(-1)^{s}}{2}\right]\left(z \cdot \partial_{y}+z \cdot \partial_{\bar{y}}\right)^{s} C_{s}^{\left(\frac{d-3}{2}\right)}\left(\frac{z \cdot \partial_{y}-z \cdot \partial_{\bar{y}}}{z \cdot \partial_{y}+z \cdot \partial_{\bar{y}}}\right) \\
& \times\left.\int_{0}^{\infty}\left(\prod_{i=1}^{3} \frac{d t_{i}}{t_{i}} t_{i}^{\frac{d}{2}-1}\right) e^{-t_{1}\left(y-x_{1}\right)^{2}-t_{2}\left(\bar{y}-x_{2}\right)^{2}-t_{3}\left(x_{1}-x_{2}\right)^{2}}\right|_{y, \bar{y} \rightarrow y_{3}} .
\end{aligned}
$$

Hint: Express the two-point function of the fundamental scalar in Schwinger-parameterised form

$$
\left\langle\phi^{a}\left(y_{1}\right) \phi^{b}\left(y_{2}\right)\right\rangle=\frac{\delta^{a b}}{\Gamma\left(\frac{d}{2}-1\right)} \int_{0}^{\infty} \frac{d t}{t} t^{\frac{d}{2}-1} e^{-t y_{12}^{2}} .
$$

By extracting the coefficient of $\left(y_{13} \cdot z\right)^{s}$ in (4.80), confirm the expression (4.79) for the overall coefficient of the three-point function.

Likewise, two-point functions are also fixed up to an overall coefficient by conformal symmetry

$$
\left\langle\mathscr{J}_{s}\left(y_{1} ; z_{1}\right) \mathscr{J}_{s}\left(y_{2} ; z_{2}\right)\right\rangle=\frac{C_{\mathscr{J}_{s}}}{\left(y_{12}^{2}\right)^{s+d-2}}\left(z_{1} \cdot z_{2}+\frac{2 z_{1} \cdot y_{12} z_{2} \cdot y_{21}}{y_{12}^{2}}\right)^{s} .
$$

A similar exercise in Wick contractions yields,

$$
\mathrm{C}_{\mathscr{J}_{s}}=2^{s+1} N\left[\frac{1+(-1)^{s}}{2}\right] \frac{(d-3)_{s}(d-3)_{2 s}}{\Gamma(s+1)} .
$$

\subsubsection{Holographic reconstruction}

Before we proceed to extract the cubic couplings $g_{0,0, s}$ holographically, let us emphasise that the GKP/W formula (2.5) is only meaningful if the two-point function normalisations in both the bulk and boundary are consistent. In the following we employ the unit normalisation ${ }^{38}$

$$
\left\langle\mathscr{J}_{s}\left(y_{1} ; z_{1}\right) \mathscr{J}_{s}\left(y_{2} ; z_{2}\right)\right\rangle=\frac{1}{\left(y_{12}^{2}\right)^{s+d-2}}\left(z_{1} \cdot z_{2}+\frac{2 z_{1} \cdot y_{12} z_{2} \cdot y_{21}}{y_{12}^{2}}\right)^{s} .
$$

To extract the cubic coupling we compare the bulk (4.77) and boundary (4.78) results with normalisation (4.84), to obtain $[33]^{39}$

\footnotetext{
${ }^{38}$ On the bulk side this entails sending $\mathscr{J}_{s} \rightarrow \frac{1}{\sqrt{C_{s+d-2, s}}} \mathscr{J}_{s}$, while in the CFT we send $\mathscr{J}_{s} \rightarrow \frac{1}{\sqrt{C_{\mathscr{f}_{s}}}} \mathscr{J}_{s}$.

${ }^{39}$ See also [36] for the earlier $s=0$ case on $\mathrm{AdS}_{4}$. The vertex in this case is in fact vanishing, which can be seen by inserting $d=3$ and $s=0$ in (4.85). To reconcile this result with the non-zero dual CFT correlator $\langle\mathscr{O} \mathscr{O} \mathscr{O}\rangle$, in this case one may add a boundary term to the bulk action which generates the CFT result. This was carried out [162] for the duality with four-dimensional $\mathscr{N}=8$ gauged supergravity [163] in the bulk, which has no $A^{3}$ cubic couplings but the dual CFT correlators are non-vanishing.
} 


$$
g_{0,0, s}=\frac{2^{\frac{3 d-s-1}{2}} \pi^{\frac{d-3}{4}} \Gamma\left(\frac{d-1}{2}\right) \sqrt{\Gamma\left(s+\frac{d}{2}-\frac{1}{2}\right)}}{\sqrt{N} \sqrt{s !} \Gamma(d+s-3)} .
$$

Holography therefore indicates that the sector of a would-be cubic order action on $\operatorname{AdS}_{d+1}$ involving interactions of the gauge fields with two scalars (on-shell) takes the form

$$
\begin{aligned}
& \frac{1}{G} S_{\mathrm{HS} \text { AdS }}\left[\varphi_{s}\right] \\
& \quad=\sum_{s \in 2 \mathbb{Z}} \int_{\operatorname{AdS}_{d+1}} \frac{1}{2} \varphi^{\mu_{1} \ldots \mu_{s}}(x)\left(\square-m_{s}^{2}\right) \varphi_{\mu_{1} \ldots \mu_{s}}(x)+g_{0,0, s} 2^{s} \varphi_{\mu_{1} \ldots \mu_{s}} \varphi_{0}(x) \nabla^{\mu_{1}} \ldots \nabla^{\mu_{s}} \varphi_{0}(x)+\ldots,
\end{aligned}
$$

with $m_{s}^{2}=(s-2)(s+d-2)$.

A few concluding comments:

- In obtaining the result (4.86), we did not employ any notion of higher-spin gauge symmetry - under which the theory should still be invariant at the interacting level. An important non-trivial check of the result ${ }^{40}$ was the demonstration that it would coincide with the vertex obtained purely from requiring higher-spin gauge invariance at the interacting level [164], i.e. the Noether procedure. This also served as a test of higher-spin holography itself, generalising the existing tree-level three-point function tests in $\mathrm{AdS}_{4}$ [101] and $\mathrm{AdS}_{3}$ [103,104] to generic dimensions.

- At the same time, the holographic approach to constructing higher-spin interactions appears to be more efficient than the Noether procedure. Indeed, invariance under linearised higherspin gauge transformations (1.42) is insufficient to fix the relative couplings (4.85), requiring the consideration of higher-order consistency conditions. See e.g. $[49,61,119,165]$. The holographic approach requires only knowledge of three-point free CFT correlators.

- The methods and results presented here can be straightforwardly carried over to other instances of higher-spin holography. So far, the 0-0-s cubic couplings have also been holographically reconstructed for the higher-spin theory dual to the free fermion vector model [38]. Moreover, the tools $\$ 4.1 .2$ for evaluating three-point Witten diagrams with external fields of arbitrary integer spin also apply to massive fields and can be easily extended to representations of mixed-symmetry, as relevant for string theory. In particular, these methods and results are applicable beyond higher-spin-symmetric set-ups.

- The holographic reconstruction of interactions can also in principle be executed at quartic and higher-orders. So far, there have been results for the quartic self-interaction of the scalar in the minimal bosonic higher-spin theory $[32,33,166]$. At this order the question of locality becomes important, as one is inevitably led to consider interactions with an unbounded number of derivatives. For investigations in this direction, see [33,37, 166-176].

\footnotetext{
${ }^{40}$ Together with its completion [34] for the complete action at cubic order (4.65) - i.e. for any triplet of integer spins $s_{1}-s_{2}-s_{3}$.
} 


\section{Acknowledgements}

I thank the organisers of the Twelfth Modave Summer School in Mathematical Physics for the kind invitation to present this material and, together with the other participants and lecturers, for the stimulating discussions + questions. The original results presented in the final section of these notes were obtained as part of collaborations with Xavier Bekaert, Johanna Erdmenger, Mitya Ponomarev [32,33] and Massimo Taronna [34], whom I thank for enlightening discussions over the years. I am also indebted to Massimo Taronna for constructive comments on the draft.

\section{References}

[1] E. Majorana, Relativistic theory of particles with arbitrary intrinsic angular momentum, Nuovo Cim. 9 (1932) 335-344.

[2] P. A. M. Dirac, Relativistic wave equations, Proceedings of the Royal Society of London. Series A, Mathematical and Physical Sciences 155 (1936) 447-459.

[3] M. Fierz, Force-free particles with any spin, Helv. Phys. Acta 12 (1939) 3-37.

[4] M. Fierz and W. Pauli, On relativistic wave equations for particles of arbitrary spin in an electromagnetic field, Proc. Roy. Soc. Lond. A173 (1939) 211-232.

[5] E. P. Wigner, On Unitary Representations of the Inhomogeneous Lorentz Group, Annals Math. 40 (1939) 149-204.

[6] C. Fronsdal, Massless Fields with Integer Spin, Phys. Rev. D18 (1978) 3624.

[7] J. Fang and C. Fronsdal, Massless Fields with Half Integral Spin, Phys. Rev. D18 (1978) 3630.

[8] J. Labastida and T. Morris, Massless mixed-symmetry bosonic free fields, Physics Letters B 180 (1986) $101-106$.

[9] J. M. F. Labastida, Massless bosonic free fields, Phys. Rev. Lett. 58 (Feb, 1987) 531-534.

[10] J. M. F. Labastida, Massless Particles in Arbitrary Representations of the Lorentz Group, Nucl. Phys. B322 (1989) 185-209.

[11] R. R. Metsaev, Massless mixed symmetry bosonic free fields in d-dimensional anti-de Sitter space-time, Phys. Lett. B354 (1995) 78-84.

[12] A. Y. Segal, Conformal higher spin theory, Nucl. Phys. B664 (2003) 59-130, [hep-th/0207212].

[13] K. B. Alkalaev, O. V. Shaynkman and M. A. Vasiliev, On the frame - like formulation of mixed symmetry massless fields in (A)dS(d), Nucl. Phys. B692 (2004) 363-393, [hep-th/ 0311164 ].

[14] K. B. Alkalaev, Two column higher spin massless fields in AdS(d), Theor. Math. Phys. 140 (2004) 1253-1263, [hep-th/0311212].

[15] C. Germani and A. Schelpe, Interactions of higher spin fields with gravity and branes in AdS(5), Phys. Rev. D78 (2008) 036010, [0712.2243].

[16] S. Ferrara and M. Porrati, 4D Localization in Randall-Sundrum 2 Supergravity and in Vasiliev Theories, Phys. Lett. B704 (2011) 249-254, [1108.1993].

[17] M. Kulaxizi and R. Rahman, Higher-Spin Modes in a Domain-Wall Universe, JHEP 10 (2014) 193, [1409.1942]. 
[18] R. R. Metsaev, Arbitrary spin conformal fields in (A)dS, Nucl. Phys. B885 (2014) 734-771, [1404.3712].

[19] T. Nutma and M. Taronna, On conformal higher spin wave operators, JHEP 06 (2014) 066, [1404.7452].

[20] R. Rahman and M. Taronna, Comments on Higher-Spin Fields in Nontrivial Backgrounds, in Proceedings, International Workshop on Higher Spin Gauge Theories: Singapore, Singapore, November 4-6, 2015, pp. 381-390, 2017. 1603.03050 . DOI.

[21] X. Bekaert, N. Boulanger and P. Sundell, How higher-spin gravity surpasses the spin two barrier: no-go theorems versus yes-go examples, Rev. Mod. Phys. 84 (2012) 987-1009, [1 007.0435$].$

[22] X. O. Camanho, J. D. Edelstein, J. Maldacena and A. Zhiboedov, Causality Constraints on Corrections to the Graviton Three-Point Coupling, JHEP 02 (2016) 020, [1 407 . 55 97].

[23] G. D’Appollonio, P. Di Vecchia, R. Russo and G. Veneziano, Regge behavior saves String Theory from causality violations, JHEP 05 (2015) 144, [1502.01254].

[24] D. J. Gross, High-Energy Symmetries of String Theory, Phys. Rev. Lett. 60 (1988) 1229.

[25] J. M. Maldacena, The Large N limit of superconformal field theories and supergravity, Int. J. Theor. Phys. 38 (1999) 1113-1133, [hep-th/9711200].

[26] S. S. Gubser, I. R. Klebanov and A. M. Polyakov, Gauge theory correlators from noncritical string theory, Phys. Lett. B428 (1998) 105-114, [hep-th/9802109].

[27] E. Witten, Anti-de Sitter space and holography, Adv. Theor. Math. Phys. 2 (1998) 253-291, [hep-th/9802150].

[28] P. Haggi-Mani and B. Sundborg, Free large N supersymmetric Yang-Mills theory as a string theory, JHEP 04 (2000) 031, [hep-th/ 0002189 ].

[29] E. Witten, Stringy gravity, interacting tensionless strings and massless higher spins, .

[30] E. Sezgin and P. Sundell, Massless higher spins and holography, Nucl. Phys. B644 (2002) 303-370, [hep-th/0205131].

[31] I. R. Klebanov and A. M. Polyakov, AdS dual of the critical $O(N)$ vector model, Phys. Lett. B550 (2002) 213-219, [hep-th/0210114].

[32] X. Bekaert, J. Erdmenger, D. Ponomarev and C. Sleight, Towards holographic higher-spin interactions: Four-point functions and higher-spin exchange, JHEP 03 (2015) 170, [1412. 0016 ].

[33] X. Bekaert, J. Erdmenger, D. Ponomarev and C. Sleight, Quartic AdS Interactions in Higher-Spin Gravity from Conformal Field Theory, JHEP 11 (2015) 149, [1508. 042 92].

[34] C. Sleight and M. Taronna, Higher Spin Interactions from Conformal Field Theory: The Complete Cubic Couplings, Phys. Rev. Lett. 116 (2016) 181602, [1603.00022].

[35] C. Sleight, Interactions in Higher-Spin Gravity: a Holographic Perspective. PhD thesis, Munich U., 2016. 1610.01318

[36] A. C. Petkou, Evaluating the AdS dual of the critical $O(N)$ vector model, JHEP 03 (2003) 049, [hep-th/0302063].

[37] E. D. Skvortsov and M. Taronna, On Locality, Holography and Unfolding, JHEP 11 (2015) 044, [1508.04764]. 
[38] E. D. Skvortsov, On (Un)Broken Higher-Spin Symmetry in Vector Models, in Proceedings, International Workshop on Higher Spin Gauge Theories: Singapore, Singapore, November 4-6, 2015, pp. 103-137, 2017. 1512.05994. DOI.

[39] P. A. M. Dirac, Wave equations in conformal space, Annals Math. 37 (1936) 429-442.

[40] P. Breitenlohner and D. Z. Freedman, Positive Energy in anti-De Sitter Backgrounds and Gauged Extended Supergravity, Phys. Lett. B115 (1982) 197-201.

[41] D. Sorokin, Introduction to the classical theory of higher spins, AIP Conf. Proc. 767 (2005) 172-202, [hep-th/0405069].

[42] R. Rahman, Higher Spin Theory - Part I, PoS ModaveVIII (2012) 004, [1307. 3199 ].

[43] R. Rahman and M. Taronna, From Higher Spins to Strings: A Primer, 1512.07932.

[44] A. Pashnev and M. M. Tsulaia, Dimensional reduction and BRST approach to the description of a Regge trajectory, Mod. Phys. Lett. A12 (1997) 861-870, [hep-th/9703010].

[45] A. Pashnev and M. Tsulaia, Description of the higher massless irreducible integer spins in the BRST approach, Mod. Phys. Lett. A13 (1998) 1853-1864, [hep-th/9803207].

[46] C. Burdik, A. Pashnev and M. Tsulaia, The Lagrangian description of representations of the Poincare group, Nucl. Phys. Proc. Suppl. 102 (2001) 285-292, [hep-th/0103143].

[47] D. Francia and A. Sagnotti, Free geometric equations for higher spins, Phys. Lett. B543 (2002) 303-310, [hep-th/0207002].

[48] D. Francia and A. Sagnotti, On the geometry of higher spin gauge fields, Class. Quant. Grav. 20 (2003) S473-S486, [hep-th/0212185].

[49] E. S. Fradkin and M. A. Vasiliev, Cubic Interaction in Extended Theories of Massless Higher Spin Fields, Nucl. Phys. B291 (1987) 141-171.

[50] E. S. Fradkin and M. A. Vasiliev, On the Gravitational Interaction of Massless Higher Spin Fields, Phys. Lett. B189 (1987) 89-95.

[51] R. Manvelyan and W. Ruhl, Conformal coupling of higher spin gauge fields to a scalar field in AdS(4) and generalized Weyl invariance, Phys. Lett. B593 (2004) 253-261, [hep-th/ 0403241$].$

[52] A. Fotopoulos, N. Irges, A. C. Petkou and M. Tsulaia, Higher-Spin Gauge Fields Interacting with Scalars: The Lagrangian Cubic Vertex, JHEP 10 (2007) 021, [0 708 . 1399].

[53] N. Boulanger, S. Leclercq and P. Sundell, On The Uniqueness of Minimal Coupling in Higher-Spin Gauge Theory, JHEP 08 (2008) 056, [0805.2764].

[54] Yu. M. Zinoviev, On spin 3 interacting with gravity, Class. Quant. Grav. 26 (2009) 035022, [0805.2226].

[55] R. Manvelyan and K. Mkrtchyan, Conformal invariant interaction of a scalar field with the higher spin field in AdS(D), Mod. Phys. Lett. A25 (2010) 1333-1348, [0 903.0058 ].

[56] A. Fotopoulos and M. Tsulaia, Current Exchanges for Reducible Higher Spin Modes on AdS, in 19th International Colloquium on Integrable Systems and Quantum Symmetries (ISQS-19) Prague, Czech Republic, June 17-19, 2010, 2010. 1007.0747.

[57] X. Bekaert and E. Meunier, Higher spin interactions with scalar matter on constant curvature spacetimes: conserved current and cubic coupling generating functions, JHEP 11 (2010) 116, [1007.4384]. 
[58] K. Alkalaev, FV-type action for AdS 5 mixed-symmetry fields, JHEP 03 (2011) 031, [1 011.6109$].$

[59] N. Boulanger, E. D. Skvortsov and Yu. M. Zinoviev, Gravitational cubic interactions for a simple mixed-symmetry gauge field in AdS and flat backgrounds, J. Phys. A44 (2011) 415403, [1107.1872].

[60] N. Boulanger and E. D. Skvortsov, Higher-spin algebras and cubic interactions for simple mixed-symmetry fields in AdS spacetime, JHEP 09 (2011) 063, [1107. 5028].

[61] M. A. Vasiliev, Cubic Vertices for Symmetric Higher-Spin Gauge Fields in $(A) d S_{d}$, Nucl. Phys. B862 (2012) 341-408, [1108.5921].

[62] E. Joung and M. Taronna, Cubic interactions of massless higher spins in (A)dS: metric-like approach, Nucl. Phys. B861 (2012) 145-174, [1110.5918].

[63] E. Joung, L. Lopez and M. Taronna, On the cubic interactions of massive and partially-massless higher spins in (A)dS, JHEP 07 (2012) 041, [1203. 6578].

[64] E. Joung, L. Lopez and M. Taronna, Solving the Noether procedure for cubic interactions of higher spins in (A)dS, J. Phys. A46 (2013) 214020, [1207.5520].

[65] M. Taronna, Higher-Spin Interactions: three-point functions and beyond. PhD thesis, Pisa, Scuola Normale Superiore, 2012. 1209.5755.

[66] E. Joung, L. Lopez and M. Taronna, Generating functions of (partially-)massless higher-spin cubic interactions, JHEP 01 (2013) 168, [1211.5912].

[67] N. Boulanger, D. Ponomarev and E. D. Skvortsov, Non-abelian cubic vertices for higher-spin fields in anti-de Sitter space, JHEP 05 (2013) 008, [1211. 6979].

[68] L. Lopez, On cubic AdS interactions of mixed-symmetry higher spins, TSPU Bulletin 2012 (2012) 113-118, [1210.0554].

[69] E. Joung, M. Taronna and A. Waldron, A Calculus for Higher Spin Interactions, JHEP 07 (2013) $186,[1305.5809]$.

[70] O. Aharony, S. S. Gubser, J. M. Maldacena, H. Ooguri and Y. Oz, Large N field theories, string theory and gravity, Phys. Rept. 323 (2000) 183-386, [hep-th/9905111].

[71] A. Zaffaroni, Introduction to the AdS-CFT correspondence, Class. Quant. Grav. 17 (2000) 3571-3597.

[72] E. D'Hoker and D. Z. Freedman, Supersymmetric gauge theories and the AdS / CFT correspondence, in Strings, Branes and Extra Dimensions: TASI 2001: Proceedings, pp. 3-158, 2002. hep-th/0201253.

[73] J. Polchinski, Introduction to Gauge/Gravity Duality, in Proceedings, Theoretical Advanced Study Institute in Elementary Particle Physics (TASI 2010). String Theory and Its Applications: From meV to the Planck Scale: Boulder, Colorado, USA, June 1-25, 2010, pp. 3-46, 2010. 1010.6134 . DOI.

[74] V. E. Hubeny, The AdS/CFT Correspondence, Class. Quant. Grav. 32 (2015) 124010, [1501.00007].

[75] H. Nastase, Introduction to the ADS/CFT Correspondence. Cambridge University Press, Cambridge, 2015.

[76] M. Ammon and J. Erdmenger, Gauge/gravity duality. Cambridge Univ. Pr., Cambridge, UK, 2015. 
[77] I. R. Klebanov and E. Witten, AdS / CFT correspondence and symmetry breaking, Nucl. Phys. B556 (1999) 89-114, [hep-th/9905104].

[78] S. Lee, S. Minwalla, M. Rangamani and N. Seiberg, Three point functions of chiral operators in D = 4, N=4 SYM at large N, Adv. Theor. Math. Phys. 2 (1998) 697-718, [hep-th/9806074].

[79] W. Mueck and K. S. Viswanathan, Conformal field theory correlators from classical scalar field theory on AdS(d+1), Phys. Rev. D58 (1998) 041901, [hep-th/ 9804035 ].

[80] W. Mueck and K. S. Viswanathan, Conformal field theory correlators from classical field theory on anti-de Sitter space. 2. Vector and spinor fields, Phys. Rev. D58 (1998) 106006, [hep-th/9805145].

[81] D. Z. Freedman, S. D. Mathur, A. Matusis and L. Rastelli, Comments on 4 point functions in the CFT / AdS correspondence, Phys. Lett. B452 (1999) 61-68, [hep-th/9808006].

[82] G. Chalmers and K. Schalm, The Large N(c) limit of four point functions in N=4 superYang-Mills theory from Anti-de Sitter supergravity, Nucl. Phys. B554 (1999) 215-236, [hep-th/9810051].

[83] H. Liu and A. A. Tseytlin, On four point functions in the CFT / AdS correspondence, Phys. Rev. D59 (1999) 086002, [hep-th/9807097].

[84] E. D'Hoker and D. Z. Freedman, General scalar exchange in AdS(d+1), Nucl. Phys. B550 (1999) 261-288, [hep-th/9811257].

[85] E. D’Hoker and D. Z. Freedman, Gauge boson exchange in AdS(d+1), Nucl. Phys. B544 (1999) 612-632, [hep-th/9809179].

[86] H. Liu and A. A. Tseytlin, $D=4$ superYang-Mills, $D=5$ gauged supergravity, and $D=4$ conformal supergravity, Nucl. Phys. B533 (1998) 88-108, [hep-th/9804083].

[87] H. Liu, Scattering in anti-de Sitter space and operator product expansion, Phys. Rev. D60 (1999) 106005, [hep-th/9811152].

[88] E. D’Hoker, D. Z. Freedman, S. D. Mathur, A. Matusis and L. Rastelli, Graviton exchange and complete four point functions in the AdS / CFT correspondence, Nucl. Phys. B562 (1999) 353-394, [hep-th/9903196].

[89] E. D'Hoker, D. Z. Freedman, S. D. Mathur, A. Matusis and L. Rastelli, Extremal correlators in the AdS / CFT correspondence, hep-th/9908160.

[90] J. Erdmenger and M. Perez-Victoria, Nonrenormalization of next-to-extremal correlators in $N=4$ SYM and the AdS / CFT correspondence, Phys. Rev. D62 (2000) 045008, [hep-th/9912250].

[91] G. Arutyunov and S. Frolov, Three point Green function of the stress energy tensor in the AdS / CFT correspondence, Phys. Rev. D60 (1999) 026004, [hep-th/9901121].

[92] G. Arutyunov and S. Frolov, Four point functions of lowest weight CPOs in N=4 SYM(4) in supergravity approximation, Phys. Rev. D62 (2000) 064016, [hep-th/ 0002170 ].

[93] M. Bianchi and A. Marchetti, Holographic three point functions: One step beyond the tradition, Nucl. Phys. B686 (2004) 261-284, [hep-th/ 0302019 ].

[94] M. Bianchi, M. Prisco and W. Mueck, New results on holographic three point functions, JHEP 11 (2003) 052, [hep-th/0310129].

[95] L. I. Uruchurtu, AdS/CFT for Four-Point Amplitudes involving Gravitino Exchange, JHEP 09 (2007) 086, [0 707.0424$]$. 
[96] L. I. Uruchurtu, Four-point correlators with higher weight superconformal primaries in the AdS/CFT Correspondence, JHEP 03 (2009) 133, [0811 . 2320].

[97] J. Penedones, Writing CFT correlation functions as AdS scattering amplitudes, JHEP 03 (2011) 025, [1011.1485].

[98] L. I. Uruchurtu, Next-next-to-extremal Four Point Functions of N=4 1/2 BPS Operators in the AdS/CFT Correspondence, JHEP 08 (2011) 133, [1106.0630].

[99] M. F. Paulos, Towards Feynman rules for Mellin amplitudes, JHEP 10 (2011) 074, [11 07.1504 ].

[100] D. Francia, J. Mourad and A. Sagnotti, (A)dS exchanges and partially-massless higher spins, Nucl. Phys. B804 (2008) 383-420, [0803.3832].

[101] S. Giombi and X. Yin, Higher Spin Gauge Theory and Holography: The Three-Point Functions, JHEP 09 (2010) 115, [0912.3462].

[102] S. Giombi and X. Yin, Higher Spins in AdS and Twistorial Holography, JHEP 04 (2011) 086, [1004.3736].

[103] C.-M. Chang and X. Yin, Higher Spin Gravity with Matter in AdS 3 and Its CFT Dual, JHEP 10 (2012) 024, [1106.2580].

[104] M. Ammon, P. Kraus and E. Perlmutter, Scalar fields and three-point functions in D=3 higher spin gravity, JHEP 07 (2012) 113, [1111.3926].

[105] E. Hijano, P. Kraus and E. Perlmutter, Matching four-point functions in higher spin $A d S_{3} / C F T_{2}$, JHEP 05 (2013) 163, [1302.6113].

[106] M. S. Costa, V. Gonçalves and J. Penedones, Spinning AdS Propagators, JHEP 09 (2014) 064, [1404.5625].

[107] S. Giombi and I. R. Klebanov, One Loop Tests of Higher Spin AdS/CFT, JHEP 12 (2013) 068, [1308.2337].

[108] S. Giombi, I. R. Klebanov and B. R. Safdi, Higher Spin AdS $S_{d+1} / C F T_{d}$ at One Loop, Phys. Rev. D89 (2014) 084004, [1401.0825].

[109] S. Giombi, I. R. Klebanov and A. A. Tseytlin, Partition Functions and Casimir Energies in Higher Spin AdS $S_{d+1} / C F T_{d}$, Phys. Rev. D90 (2014) 024048, [1402.5396].

[110] M. Beccaria and A. A. Tseytlin, Higher spins in AdS 5 at one loop: vacuum energy, boundary conformal anomalies and AdS/CFT, JHEP 11 (2014) 114, [1410.3273].

[111] T. Basile, X. Bekaert and N. Boulanger, Flato-Fronsdal theorem for higher-order singletons, JHEP 11 (2014) 131, [1410.7668].

[112] Y. Pang, E. Sezgin and Y. Zhu, One Loop Tests of Supersymmetric Higher Spin AdS $4 / C F T_{3}$, Phys. Rev. D95 (2017) 026008, [1608.07298].

[113] J.-B. Bae, E. Joung and S. Lal, One-loop test of free SU(N) adjoint model holography, JHEP 04 (2016) 061, [1603.05387].

[114] J.-B. Bae, E. Joung and S. Lal, On the Holography of Free Yang-Mills, JHEP 10 (2016) 074, [1607.07651].

[115] M. GÃijnaydin, E. D. Skvortsov and T. Tran, Exceptional F(4) higher-spin theory in AdS 6 at one-loop and other tests of duality, JHEP 11 (2016) 168, [1608.07582]. 
[116] J.-B. Bae, E. Joung and S. Lal, One-Loop Free Energy of Tensionless Type IIB String in AdS $S_{5} \times S^{5}$, 1701.01507.

[117] D. Simmons-Duffin, TASI Lectures on the Conformal Bootstrap, 1602.07982.

[118] J. Maldacena and A. Zhiboedov, Constraining Conformal Field Theories with A Higher Spin Symmetry, J. Phys. A46 (2013) 214011, [1112.1016].

[119] N. Boulanger, D. Ponomarev, E. D. Skvortsov and M. Taronna, On the uniqueness of higher-spin symmetries in AdS and CFT, Int. J. Mod. Phys. A28 (2013) 1350162, [1305. 5180 ].

[120] V. Alba and K. Diab, Constraining conformal field theories with a higher spin symmetry in $d=4$, 1307.8092 .

[121] V. Alba and K. Diab, Constraining conformal field theories with a higher spin symmetry in d $>3$ dimensions, JHEP 03 (2016) 044, [1510.02535].

[122] D. Friedan and C. A. Keller, Cauchy Conformal Fields in Dimensions d > 2, Commun. Math. Phys. 348 (2016) 655-694, [1509.07475].

[123] N. S. Craigie, V. K. Dobrev and I. T. Todorov, Conformally Covariant Composite Operators in Quantum Chromodynamics, Annals Phys. 159 (1985) 411-444.

[124] M. A. Vasiliev, Nonlinear equations for symmetric massless higher spin fields in (A)dS(d), Phys. Lett. B567 (2003) 139-151, [hep-th/0304049].

[125] M. A. Vasiliev, Consistent equation for interacting gauge fields of all spins in $(3+1)$-dimensions, Phys. Lett. B243 (1990) 378-382.

[126] N. Boulanger and P. Sundell, An action principle for Vasiliev's four-dimensional higher-spin gravity, J. Phys. A44 (2011) 495402, [1102.2219].

[127] N. Boulanger, N. Colombo and P. Sundell, A minimal BV action for Vasiliev's four-dimensional higher spin gravity, JHEP 10 (2012) 043, [1205.3339].

[128] V. E. Didenko, N. G. Misuna and M. A. Vasiliev, Charges in nonlinear higher-spin theory, 1512.07626.

[129] X. Bekaert, S. Cnockaert, C. Iazeolla and M. A. Vasiliev, Nonlinear higher spin theories in various dimensions, in Higher spin gauge theories: Proceedings, 1st Solvay Workshop: Brussels, Belgium, 12-14 May, 2004, pp. 132-197, 2004. hep-th/0503128.

[130] C. Iazeolla, On the Algebraic Structure of Higher-Spin Field Equations and New Exact Solutions. $\mathrm{PhD}$ thesis, Rome U., Tor Vergata, 2008. 0807.0406.

[131] V. E. Didenko and E. D. Skvortsov, Elements of Vasiliev theory, 1401.2975.

[132] M. Bianchi, J. F. Morales and H. Samtleben, On stringy AdS(5) x $S^{* * 5}$ and higher spin holography, JHEP 07 (2003) 062, [hep-th/ 0305052 ].

[133] N. Beisert, M. Bianchi, J. F. Morales and H. Samtleben, On the spectrum of AdS / CFT beyond supergravity, JHEP 02 (2004) 001, [hep-th/ 03102 92].

[134] N. Beisert, M. Bianchi, J. F. Morales and H. Samtleben, Higher spin symmetry and N=4 SYM, JHEP 07 (2004) 058, [hep-th/ 0405057 ].

[135] M. Bianchi, Higher spin symmetry (breaking) in N=4 sym theory and holography, Comptes Rendus Physique 5 (2004) 1091-1099, [hep-th/ 0409292$].$ 
[136] M. Bianchi, Higher spins and stringy AdS(5) x S**5, Fortsch. Phys. 53 (2005) 665-691, [hep-th/0409304].

[137] M. Bianchi and V. Didenko, Massive higher spin multiplets and holography, in Higher spin gauge theories: Proceedings, 1st Solvay Workshop: Brussels, Belgium, 12-14 May, 2004, pp. 1-20, 2004. hep-th/0502220.

[138] M. Bianchi, P. J. Heslop and F. Riccioni, More on La Grande Bouffe, JHEP 08 (2005) 088, [hep-th/0504156].

[139] M. Bianchi and F. Riccioni, Massive higher spins and holography, J. Phys. Conf. Ser. 33 (2006) 49-56, [hep-th/0601071].

[140] C.-M. Chang, S. Minwalla, T. Sharma and X. Yin, ABJ Triality: from Higher Spin Fields to Strings, J. Phys. A46 (2013) 214009, [1207.4485].

[141] M. R. Gaberdiel and R. Gopakumar, Higher Spins \& Strings, JHEP 11 (2014) 044, [1 406.6103$].$

[142] M. R. Gaberdiel and R. Gopakumar, Stringy Symmetries and the Higher Spin Square, J. Phys. A48 (2015) 185402, [1501.07236].

[143] M. R. Gaberdiel, C. Peng and I. G. Zadeh, Higgsing the stringy higher spin symmetry, JHEP 10 (2015) 101, [1506.02045].

[144] M. R. Gaberdiel and R. Gopakumar, String Theory as a Higher Spin Theory, JHEP 09 (2016) 085, [1512.07237].

[145] R. G. Leigh and A. C. Petkou, Holography of the N=1 higher spin theory on AdS(4), JHEP 06 (2003) 011, [hep-th/0304217].

[146] E. Sezgin and P. Sundell, Holography in 4D (super) higher spin theories and a test via cubic scalar couplings, JHEP 07 (2005) 044, [hep-th/ 030504 0].

[147] S. Giombi, S. Minwalla, S. Prakash, S. P. Trivedi, S. R. Wadia and X. Yin, Chern-Simons Theory with Vector Fermion Matter, Eur. Phys. J. C72 (2012) 2112, [1110.4386].

[148] S. Giombi and X. Yin, The Higher Spin/Vector Model Duality, J. Phys. A46 (2013) 214003, $[1208.4036]$.

[149] S. Giombi, TASI Lectures on the Higher Spin - CFT duality, 1607.02967.

[150] G. Mack and A. Salam, Finite component field representations of the conformal group, Annals Phys. 53 (1969) 174-202.

[151] C. Fronsdal, Singletons and Massless, Integral Spin Fields on de Sitter Space (Elementary Particles in a Curved Space. 7., Phys. Rev. D20 (1979) 848-856.

[152] T. Biswas and W. Siegel, Radial dimensional reduction: Anti-de Sitter theories from flat, JHEP 07 (2002) 005, [hep-th/0203115].

[153] S. Weinberg, Six-dimensional Methods for Four-dimensional Conformal Field Theories, Phys. Rev. D82 (2010) 045031, [1006.3480].

[154] M. S. Costa, J. Penedones, D. Poland and S. Rychkov, Spinning Conformal Correlators, JHEP 11 (2011) 071, [1107.3554].

[155] M. Grigoriev and A. Waldron, Massive Higher Spins from BRST and Tractors, Nucl. Phys. B853 (2011) 291-326, [1104.4994]. 
[156] X. Bekaert and M. Grigoriev, Notes on the ambient approach to boundary values of AdS gauge fields, J. Phys. A46 (2013) 214008, [1207.3439].

[157] A. Mikhailov, Notes on higher spin symmetries, hep-th/0201019.

[158] R. R. Metsaev, Cubic interaction vertices of massive and massless higher spin fields, Nucl. Phys. B759 (2006) 147-201, [hep-th/0 512342].

[159] J. D. Qualls, Lectures on Conformal Field Theory, 1511.04074.

[160] S. Rychkov, EPFL Lectures on Conformal Field Theory in D>=3 Dimensions, 1601.05000.

[161] H. Osborn and A. C. Petkou, Implications of conformal invariance in field theories for general dimensions, Annals Phys. 231 (1994) 311-362, [hep-th/9307010].

[162] D. Z. Freedman, K. Pilch, S. S. Pufu and N. P. Warner, Boundary Terms and Three-Point Functions: An AdS/CFT Puzzle Resolved, 1611.01888.

[163] B. de Wit and H. Nicolai, N=8 Supergravity, Nucl. Phys. B208 (1982) 323.

[164] C. Sleight and M. Taronna, Higher-Spin Algebras, Holography and Flat Space, 1609.00991.

[165] P. Kessel, G. Lucena Gómez, E. Skvortsov and M. Taronna, Higher Spins and Matter Interacting in Dimension Three, JHEP 11 (2015) 104, [1505.05887].

[166] X. Bekaert, J. Erdmenger, D. Ponomarev and C. Sleight, Bulk quartic vertices from boundary four-point correlators, in Proceedings, International Workshop on Higher Spin Gauge Theories: Singapore, Singapore, November 4-6, 2015, pp. 291-303, 2017. 1602 . 08570 . DOI.

[167] G. Barnich and M. Henneaux, Consistent couplings between fields with a gauge freedom and deformations of the master equation, Phys. Lett. B311 (1993) 123-129, [hep-th/9304057].

[168] M. Taronna, Higher-Spin Interactions: four-point functions and beyond, JHEP 04 (2012) 029, [1107.5843].

[169] P. Dempster and M. Tsulaia, On the Structure of Quartic Vertices for Massless Higher Spin Fields on Minkowski Background, Nucl. Phys. B865 (2012) 353-375, [1203.5597].

[170] M. A. Vasiliev, Star-Product Functions in Higher-Spin Theory and Locality, JHEP 06 (2015) 031, [1502.02271].

[171] N. Boulanger, P. Kessel, E. D. Skvortsov and M. Taronna, Higher spin interactions in four-dimensions: Vasiliev versus Fronsdal, J. Phys. A49 (2016) 095402, [1508.04139].

[172] M. Taronna, Pseudo-local Theories: A Functional Class Proposal, in Proceedings, International Workshop on Higher Spin Gauge Theories: Singapore, Singapore, November 4-6, 2015, pp. 59-84, 2017. 1602.08566 . DOI.

[173] M. A. Vasiliev, Current Interactions, Locality and Holography from the 0-Form Sector of Nonlinear Higher-Spin Equations, 1605.02662.

[174] M. Taronna, A Note on Field Redefinitions and Higher-Spin Equations, EPJ Web Conf. 125 (2016) 05025, [1607.04718].

[175] M. Taronna, On the Non-Local Obstruction to Interacting Higher Spins in Flat Space, 1701.05772 .

[176] R. Roiban and A. A. Tseytlin, On four-point interactions in massless higher spin theory in flat space, 1701.05773. 